\title{
Computation of optimized arrays for 3-D electrical imaging surveys
}

\section{Journal Article}

\section{Author(s):}

Loke, M.H.; Wilkinson, P.B.; Uhlemann, S.S.; Chambers, J.E.; Oxby, L.S.

Publication date:

2014-12

Permanent link:

https://doi.org/10.3929/ethz-b-000095234

\section{Rights / license:}

In Copyright - Non-Commercial Use Permitted

\section{Originally published in:}

Geophysical Journal International 199(3), https://doi.org/10.1093/gji/ggu357 


\title{
Computation of optimized arrays for 3-D electrical imaging surveys
}

\author{
M. H. Loke, ${ }^{1}$ P. B. Wilkinson, ${ }^{2}$ S. S. Uhlemann, ${ }^{2,3}$ J. E. Chambers ${ }^{2}$ and L. S. Oxby ${ }^{2}$ \\ ${ }^{1}$ Geotomo Software, 115 Cangkat Minden Jalan 5, Minden Heights, 11700 Gelugor, Penang, Malaysia. E-mail: drmhloke@hotmail.com \\ ${ }^{2}$ British Geological Survey, Natural Environment Research Council, Kingsley Dunham Centre, Keyworth, Nottingham NG12 5GG, United Kingdom \\ ${ }^{3}$ ETH Zurich, Institute of Geophysics, Sonneggstrasse 5, CH-8092 Zurich, Switzerland
}

Accepted 2014 September 9. Received 2014 September 9; in original form 2013 December 15

\begin{abstract}
SUMMAR Y
3-D electrical resistivity surveys and inversion models are required to accurately resolve structures in areas with very complex geology where 2-D models might suffer from artefacts. Many 3-D surveys use a grid where the number of electrodes along one direction $(x)$ is much greater than in the perpendicular direction $(y)$. Frequently, due to limitations in the number of independent electrodes in the multi-electrode system, the surveys use a roll-along system with a small number of parallel survey lines aligned along the $x$-direction. The 'Compare $\mathrm{R}$ ' array optimization method previously used for 2-D surveys is adapted for such 3-D surveys. Offset versions of the inline arrays used in 2-D surveys are included in the number of possible arrays (the comprehensive data set) to improve the sensitivity to structures in between the lines. The array geometric factor and its relative error are used to filter out potentially unstable arrays in the construction of the comprehensive data set. Comparisons of the conventional (consisting of dipole-dipole and Wenner-Schlumberger arrays) and optimized arrays are made using a synthetic model and experimental measurements in a tank. The tests show that structures located between the lines are better resolved with the optimized arrays. The optimized arrays also have significantly better depth resolution compared to the conventional arrays.
\end{abstract}

Key words: Numerical solutions; Inverse theory; Electrical properties.

\section{INTRODUCTION}

2-D resistivity surveys are now widely used to map areas with moderately complex geology. These surveys have become a standard exploration tool to solve many field problems in agriculture, groundwater exploration, engineering site investigation, environmental assessment, mineral exploration and even hydrocarbon mapping (Dahlin 1996; Auken et al. 2006; Loke et al. 2013a). However, in areas with very complex geology, it has been found that the models obtained from 2-D surveys can suffer from artefacts due to offline structures (Dahlin \& Loke 1998; Bentley \& Gharibi 2004). For such problems, 3-D surveys using a rectangular grid of electrodes provide the best solution (Dahlin et al. 2002; Gharibi \& Bentley 2005; Chambers et al. 2006, 2012). Frequently, due to physical limitations in many commercial multielectrode resistivity meters systems, the number of electrode positions along one direction (the ' $x$ ' direction) is much larger than in the perpendicular (the ' $y$ ') direction. Furthermore, the distance between the lines in the $y$ direction is frequently twice (or more) the inline electrode spacing in the $x$ direction (Gharibi \& Bentley 2005). This is a common practical arrangement to cover the widest possible area with a limited number of independent electrodes.

In recent years, there has been significant progress in algorithms to automatically determine the set of array configurations that will maximize the resolution of the subsurface inversion model for 2-D surveys (Stummer et al. 2004; Wilkinson et al. 2006). The 'Compare $\mathrm{R}$ ' algorithm proved to be the best method in terms of determining arrays that have the highest resolution among the techniques that were tested (Loke et al. 2010a). A very fast version of the 'Compare $\mathrm{R}$ ' method using the microcomputer graphics processing unit (GPU) was implemented by Loke et al. (2010b). However, 3-D surveys present new challenges even for the fast GPU-based algorithm due to the larger number of electrodes in the survey grid, as well the larger number of parameters in the subsurface model.

In this paper, the mathematical background for the 'Compare $\mathrm{R}$ ' method is briefly described. We then describe a new method to generate the set of possible arrays for 3-D surveys grids where the number of electrodes along one axis is much greater than along the perpendicular direction. This is followed by plots of the model resolution for the optimized arrays and conventional arrays. Finally we present results from tests using a synthetic model and an experimental data set.

\section{THEORY}

\subsection{Data inversion, model resolution and the 'Compare $\mathbf{R}$ ' method}

The smoothness-constrained least-squares optimization method is frequently used for 2-D and 3-D inversion of resistivity data 
(deGroot-Hedlin \& Constable 1990; Ellis \& Oldenburg 1994; Loke et al. 2013a, 2014b). The 3-D model usually consists of many quadrilateral cells (Loke \& Barker 1996). The linearized leastsquares equation that gives the relationship between the model parameters (r) and the data misfit ( $(\mathbf{g})$ is

$\left(\mathbf{G}^{\mathrm{T}} \mathbf{G}+\lambda \mathbf{C}\right) \boldsymbol{\Delta} r_{i}=\mathbf{G}^{\mathbf{T}} \mathbf{g}-\lambda \mathbf{C} \mathbf{r}_{i-\mathbf{1}}$.

The Jacobian matrix $\mathbf{G}$ contains the sensitivities of the (logarithms of the) apparent resistivities with respect to the model resistivity values, $\mathbf{C}$ contains the roughness filter constraint and $\lambda$ is a damping factor. The model resolution matrix $\mathbf{R}$ (Loke et al. 2010a) is given by

$\mathbf{R}=\mathbf{B} \mathbf{A}$,

where

$\mathbf{A}=\mathbf{G}^{\mathrm{T}} \mathbf{G} \quad$ and $\quad \mathbf{B}=(\mathbf{A}+\lambda \mathbf{C})^{-1}$.

The number of possible four-electrode configurations is $N(N-$ $1)(N-2)(N-3) / 8$, where $N$ is the number of electrodes. This number is reduced by excluding the gamma type arrays with crossed current and potential electrodes (Carpenter \& Habberjam 1956) and those with large geometric factors (Stummer et al. 2004; Loke et al. 2010a). A maximum geometric factor limit is set according to the expected noise level in a field survey (Wilkinson et al. 2012). A local optimization procedure is used to select a subset of the viable configurations (the comprehensive data set) that will maximize the model resolution. A small base data set consisting of the dipoledipole configurations with a dipole length ' $a$ ' of 1 unit electrode spacing and maximum ' $n$ ' value (the ratio of the dipole separation to the dipole length) of 6-8 (depending on the maximum geometric factor set) is usually used. The change in the model resolution matrix $\Delta \mathbf{R}$ for each new configuration added to the base set is then calculated (Loke et al. 2010b) using the following equation.

$\Delta \mathbf{R}_{\mathbf{b}}=\frac{\mathbf{z}}{1+\mu}\left(\mathbf{g}^{\mathbf{T}}-\mathbf{y}^{\mathbf{T}}\right)$,

where $\mathbf{z}=\mathbf{B}_{\mathbf{b}} \mathbf{g}, \mathbf{y}=\mathbf{A}_{\mathrm{b}} \mathbf{z}$ and $\mu=$ g.z. $\mathbf{A}_{\mathrm{b}}$ and $\mathbf{B}_{\mathrm{b}}$ are the matrices, as specified in eq. (2), for the arrays in the current base data set. The vector $\mathbf{g}$ contains the sensitivity values of the model cells for the new test configuration. The following function $F_{C R}$ (Wilkinson et al. 2012) is used to the rank the improvement in the resolution of a model with $m$ cells due to an add-on array.

$F_{C R}=\frac{1}{m} \sum_{j=1}^{j=m} \frac{\Delta R_{b}(j, j)}{R_{c}(j, j)}$,

where $R_{c}$ is the comprehensive data set model resolution. A selected number of configurations that have the largest $F_{C R}$ values are added to the base set after each iteration until the target number of optimized array configurations is selected. We note that both the least-squares and array optimization methods can be modified to include the L1 and L2 norms for the model roughness and data misfit, as well as the effect of known data errors (Farquharson \& Oldenburg 1998; Loke et al. 2010b; Wilkinson et al. 2012).

\subsection{Use of the geometric factor relative error for 3-D surveys}

For surveys along 2-D lines, excluding the gamma configurations and setting a maximum limit for the geometric factor is sufficient to remove arrays that are likely to be unstable. Wilkinson et al. (2008) found that for cross-borehole surveys it was necessary to set a limit on the sensitivity of the array geometric factor to errors in the position of the electrodes as well to remove arrays that are likely to be unstable. As 3-D surveys involve cross-line measurements, we also use this method. The geometric factor $K$ for any four electrode array located on the ground surface is given by

$K=2 \pi / H, H=\left[\frac{1}{r_{A M}}-\frac{1}{r_{A N}}-\frac{1}{r_{B M}}+\frac{1}{r_{B N}}\right]$.

The current electrodes are denoted by $A$ and $B$, while the potential electrodes are $M$ and $N . r_{A M}$ is the distance between $A$ and $M$. The sensitivity $(s)$ of the geometric factor to errors in the positions of the electrodes is given by

$s^{2}=\left(\frac{\partial K}{\partial A}\right)^{2}+\left(\frac{\partial K}{\partial B}\right)^{2}+\left(\frac{\partial K}{\partial M}\right)^{2}+\left(\frac{\partial K}{\partial N}\right)^{2}$.

The sensitivity of the geometric factor to errors in the position of the $A$ electrode located at $\left(x_{A}, y_{A}\right)$ can be calculated using the following equation:

$$
\left(\frac{\partial K}{\partial A}\right)^{2}=\left(\frac{\partial K}{\partial x_{A}}\right)^{2}+\left(\frac{\partial K}{\partial y_{A}}\right)^{2} .
$$

An error in $x_{A}$ will only affect $r_{A M}$ and $r_{A N}$, thus

$\frac{\partial K}{\partial x_{A}}=-\frac{2 \pi}{H^{2}}\left(\frac{\partial H}{\partial r_{A M}} \cdot \frac{\partial r_{A M}}{\partial x_{A}}+\frac{\partial H}{\partial r_{A N}} \cdot \frac{\partial r_{A N}}{\partial x_{A}}\right)$,

where

$r_{A M}^{2}=\left(x_{A}-x_{M}\right)^{2}+\left(y_{A}-y_{M}\right)^{2}, r_{A N}^{2}=\left(x_{A}-x_{N}\right)^{2}+\left(y_{A}-y_{N}\right)^{2}$

and

$\frac{\partial H}{\partial r_{A M}}=-\frac{1}{r_{A M}^{2}}, \frac{\partial H}{\partial r_{A N}}=\frac{1}{r_{A N}^{2}}, \frac{\partial r_{A M}}{\partial x_{A}}=-2\left(x_{A}-x_{M}\right)$,

$\frac{\partial r_{A N}}{\partial x_{A}}=-2\left(x_{A}-x_{N}\right)$.

Similar equations may be derived for $y_{A}$ and the other parameters. The geometric factor relative error is then defined to be

$R_{E}=s / K$.

Following Wilkinson et al. (2008), we use a value of $5 \mathrm{~m}^{-1}$ as the cut-off factor when the unit electrode spacing is $1 \mathrm{~m}$, which gives a maximum $R_{\mathrm{E}}$ of 5 percent for a $1 \mathrm{~cm}$ uncertainty in electrode position.

\subsection{Creating the comprehensive data set for 3-D surveys}

The time taken by the 'Compare R' method is proportional to the number of arrays in the comprehensive data set $\left(n_{c}\right)$. In theory, the number of arrays in the comprehensive set is proportional to the fourth power of the number of electrodes in the survey grid. Thus, it would appear that using the 'Compare R' method would prove impractical for 3-D survey grids where the number of electrode positions often exceeds 200 that might produce a comprehensive data set with over 100 million arrays (Maurer et al. 2010). However, many commercial 3-D surveys use an arrangement where the number of electrodes along one (the ' $x$ ') direction is much larger than in the perpendicular (the ' $y$ ') direction. Furthermore, the number of electrodes in many commercial multielectrode systems is frequently very limited (less than 100). To obtain a reasonable areal coverage, a roll-along method is sometimes used. One example is shown in 


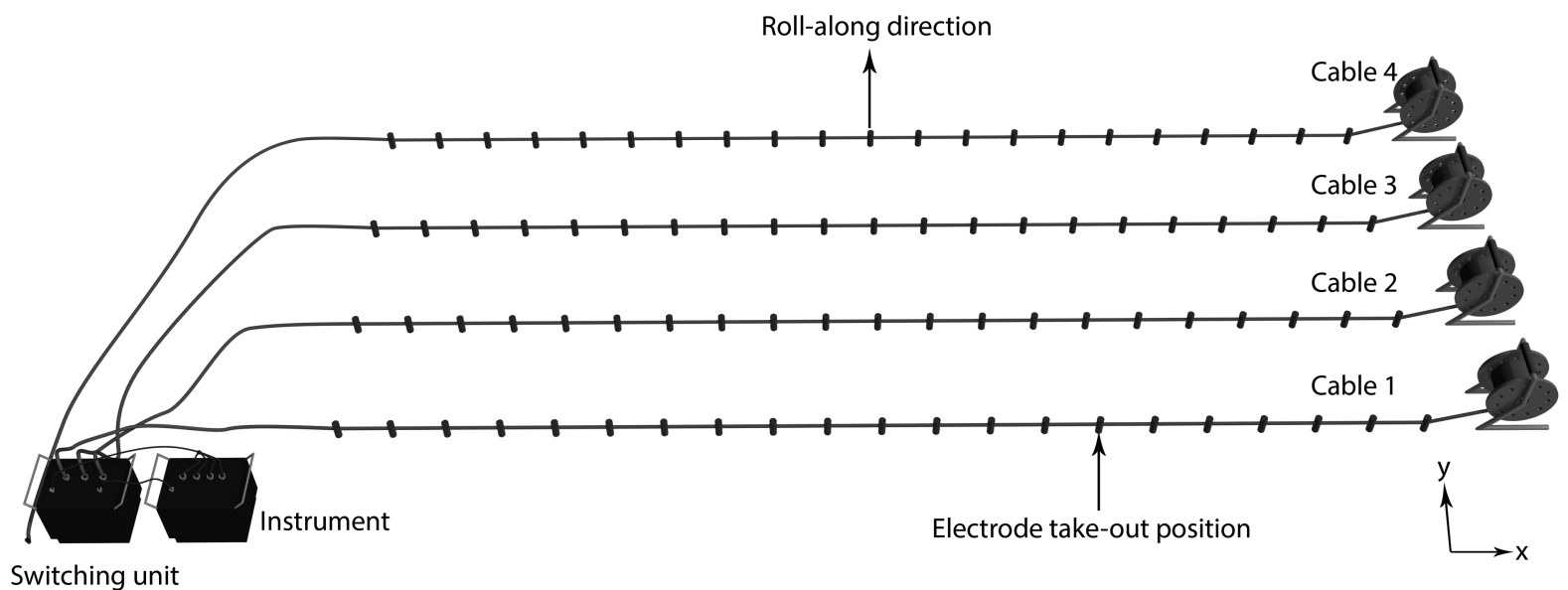

Figure 1. Arrangement of survey lines and electrodes using a three-cable system with the Abem SAS instrument.

(a)

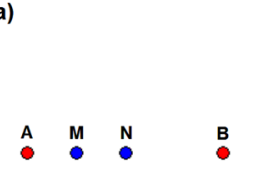

(f)<smiles>C1CC1</smiles>

(b)

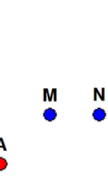

(g)

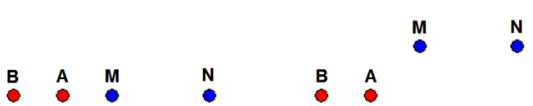

(c)

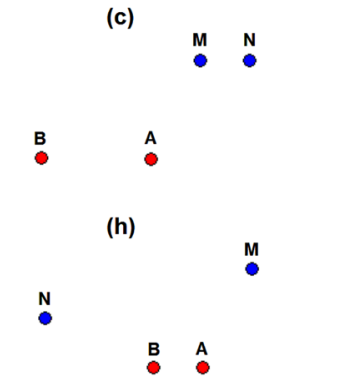

(d)

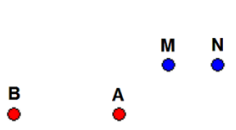

(i)

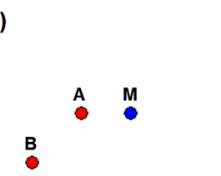

(k)

(I)

$\begin{array}{llll}B & \text { N } & \text { M } & \text { N } \\ A & \text { M } & \text { A } & \text { B }\end{array}$

Figure 2. Arrangement of alpha type array with (a) inline electrodes, (b) single offset, (c) double offset, (d) triple line and (e) quadruple line configurations. Beta type array arrangement with (f) inline electrodes, (g) single offset, (h) double offset, (i) triple line and (j) quadruple line configurations. Array configurations of the equatorial dipole-dipole type are shown in (k) and (1).

Fig. 1 where the Abem SAS system uses three cables with 21 electrodes each (Dahlin et al. 2002). While four cables are provided with the SAS system, only three of them can be connected to the resistivity meter at the same time for the 3-D electrode layout used (Fig. 1). Thus, a maximum of 63 electrodes can be addressed at a single time. However, a larger area can be surveyed by moving the setup in the $y$ direction. As an example, a $21 \times 17$ survey grid was used by Dahlin et al. (2002). Although the final survey grid has 357 electrodes positions, not all possible combinations of the electrodes can be used. Since there are only three electrodes positions in the $y$ direction in a single setup, only measurements in the $x$ direction are possible with four-electrode arrays. The method used to generate the comprehensive data set previously used for 2-D survey lines (Loke et al. 2010a) is modified for such an arrangement with several parallel lines.

The comprehensive data set for a 2-D survey line consists of all possible arrays with the viable alpha and beta four-electrode configurations (Carpenter \& Habberjam 1956; Loke et al. 2010a). Fig. 2(a) show a typical arrangement of an array of the alpha type where the potential electrodes $(M$ and $N)$ are nested between the current electrodes ( $A$ and $B$ ). For a given number of electrode positions along a survey line, all the viable alpha configurations can be easily generated. In addition to the inline alpha array, an offset version of the array where the potential electrodes are shifted to the next line is also generated (Fig. 2b). The offset version array will have a higher geometric factor than the inline array. However, if the geometric factor and geometric factor relative error (eq. 9) are less than the set limits, it is added to the comprehensive data set. A similar double offset version of the array is also generated (Fig. 2c). Fig. 2(d) shows a possible variation where the electrodes are distributed on three different lines. Similarly variations of the beta array types are shown in Figs 2(f)-(i). We also include arrays with the equatorial dipole-dipole type of configuration (Figs $2 \mathrm{k}$ and 1).

Other variations of the offset arrays were tested but they did not significantly improve the model resolution while greatly increasing the size of the comprehensive data set. One such variation is of the arrays in Figs 2(b) and (c) where $A$ and $M$ electrodes are on the same line while $N$ and $B$ are on the offset line (and a similar variation of Figs $2 \mathrm{~g}$ and $\mathrm{h}$ for the beta array). Adding these combinations increases the size of the comprehensive data set by about 57 per cent but only increases the average model resolution by less than 2 per cent. This shows that adding them will not add significant new information to that already provided by the existing configurations at the expense of significantly increasing the calculation 
(a) Inline

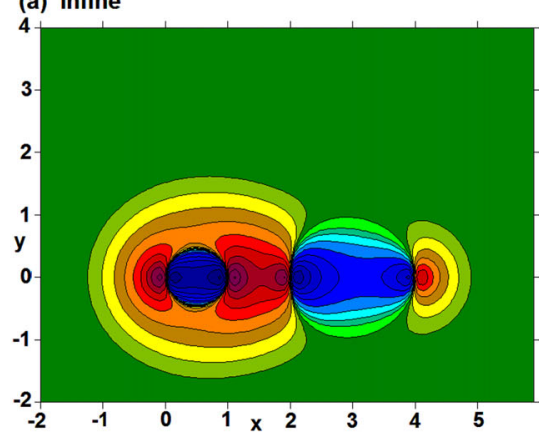

(d) Triple line

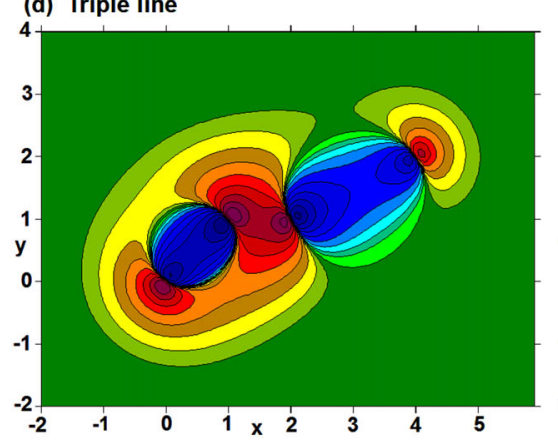

(b) Single offset

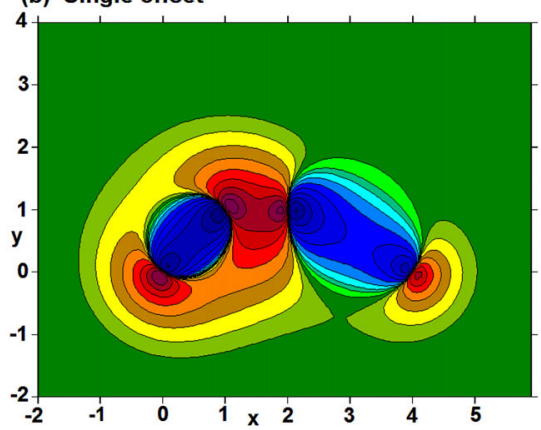

(e) Quadruple line

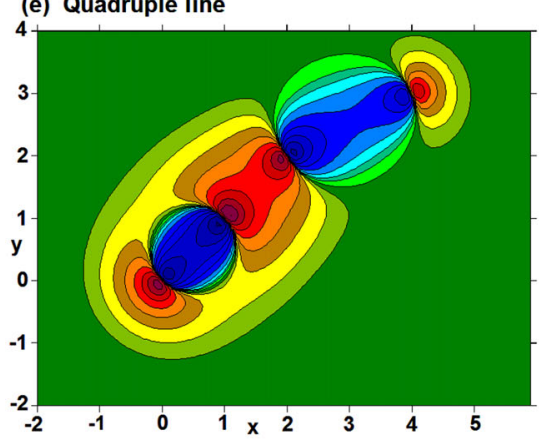

(c) Double offset

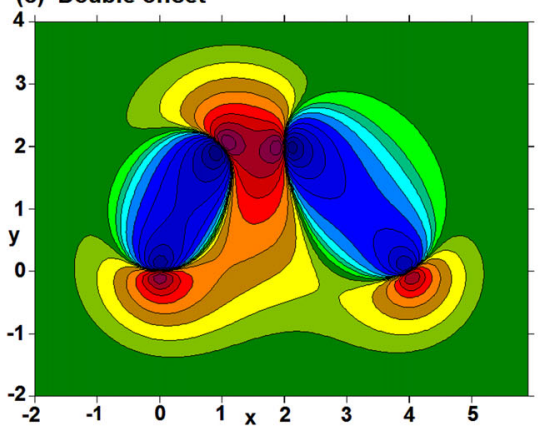

Figure 3. Sensitivity patterns at a depth of $0.1 \mathrm{~m}$ for alpha type array with (a) inline electrodes, (b) single offset, (c) double offset (d) triple line and (e) quadruple line configurations.

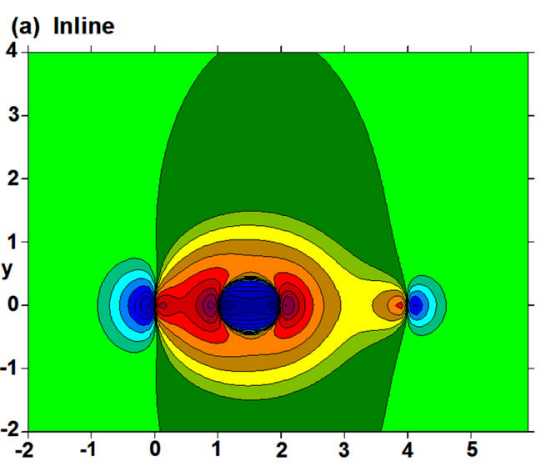

(b) Single offset

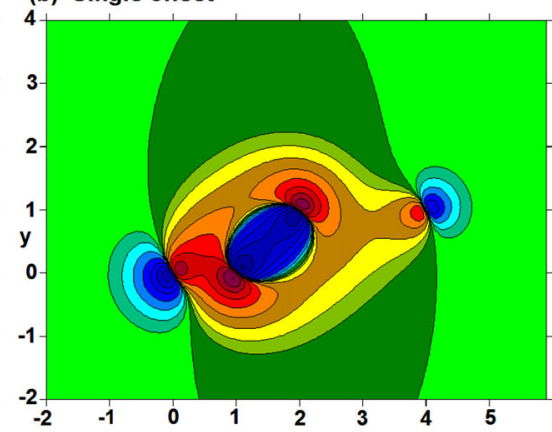

(e) Quadruple line

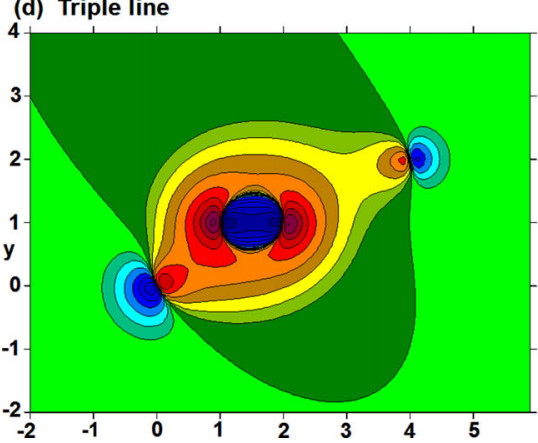

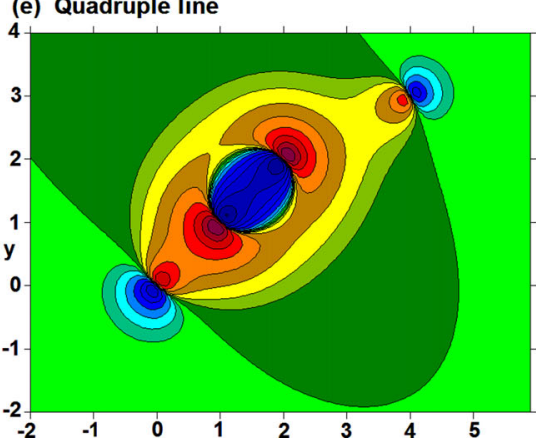

(c) Double offset
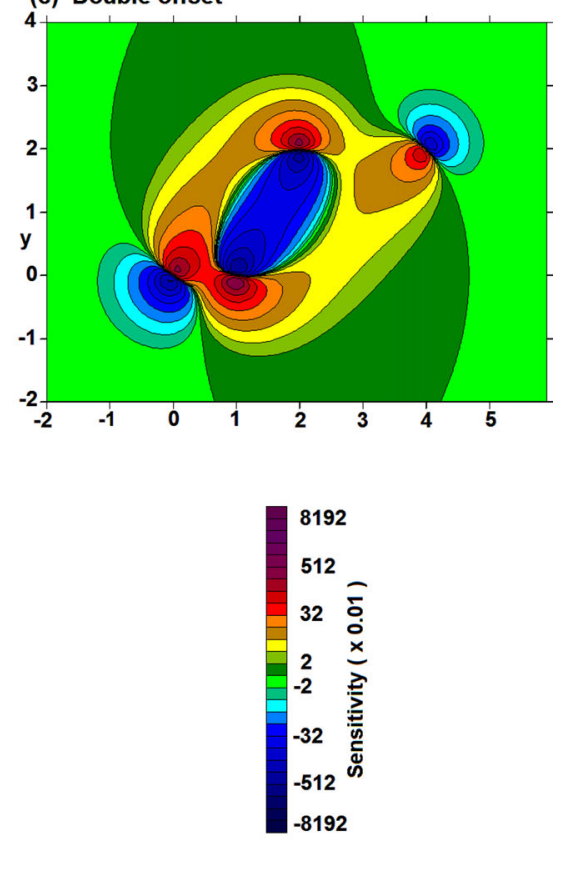

Figure 4. Sensitivity patterns at a depth of $0.1 \mathrm{~m}$ for beta type array with (a) inline electrodes, (b) single offset, (c) double offset, (d) triple line and (e) quadruple line configurations.

time. The practical use of array optimization techniques for 3-D surveys lies in a judicious selection of array types to include in the comprehensive data set that balances the improvement in the model resolution with the increase in the size of the comprehensive data set (and thus the calculation time).

The rationale for using the offset versions of the inline arrays are illustrated in Figs 3 and 4 in the form of the sensitivity patterns of the arrays at a horizontal plane with a depth of $0.1 \mathrm{~m}$. The highest positive and negative sensitivity values for the inline alpha array (Fig. 3a) are concentrated near the array axis along the $y=0$ line. The largest sensitivity values extend less than $1 \mathrm{~m}$ in the $y$ direction from the array axis. Thus, we would expect surveys carried out using only inline arrays along the $x$ direction to have significantly less sensitivity to parts of the subsurface between the lines. The 
(a) Square equatorial dipole-dipole

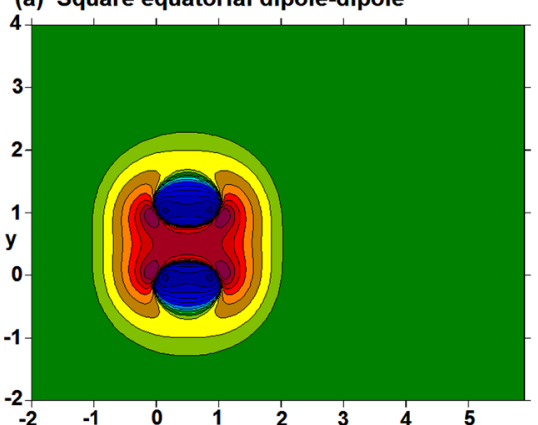

(b) Tall equatorial dipole-dipole

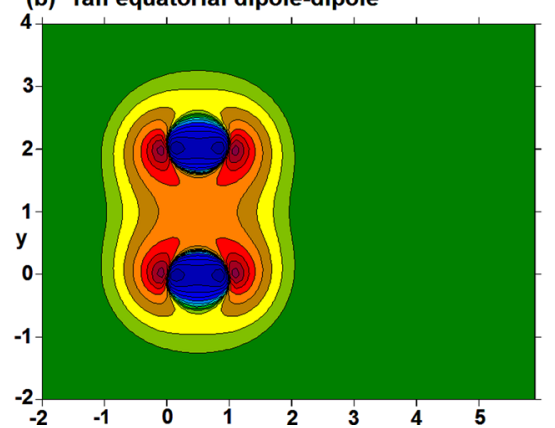

(c) Long equatorial dipole-dipole

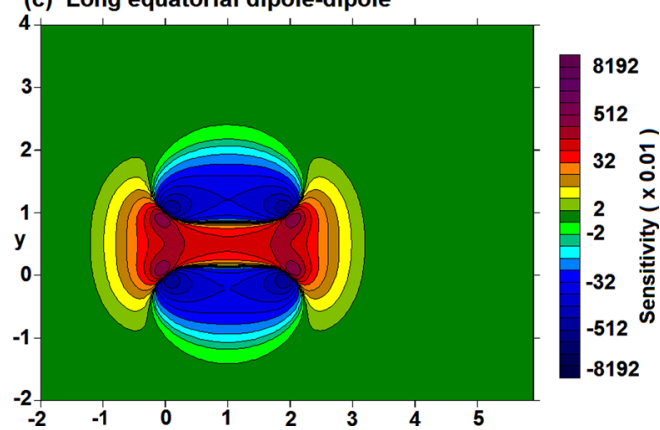

Figure 5. Sensitivity patterns at a depth of $0.1 \mathrm{~m}$ for the equatorial dipole-dipole array. (a) Square arrangement with $A-B$ dipole $1 \mathrm{~m}$ from the $M-N$ dipole. Each dipole length is $1 \mathrm{~m}$. (b) Tall version with the $A-B$ and $M-N$ dipoles of $2 \mathrm{~m}$ length placed $1 \mathrm{~m}$ apart. (c) Long version with the $A-B$ and $M-N$ dipoles $2 \mathrm{~m}$ apart.

sensitivity pattern for the alpha array with the potential electrodes offset by $1 \mathrm{~m}$ in the $y$ direction (Fig. 3b) shows much larger sensitivity values in the region between the two lines. Extending the offset to $2 \mathrm{~m}$ increases the areas with the largest sensitivity values between the $y=0$ and $y=2$ lines, although the regions with the highest sensitivity values are now less contiguous. The triple line alpha array configuration (Fig. 3d) has a fairly similar sensitivity pattern to the inline array except the sensitivity pattern is oriented in a diagonal direction covering the areas between the lines. The different versions of the beta array configuration (Fig. 4) show a similar pattern where the offset versions show larger sensitivity patterns between the lines, and the triple line configuration has a diagonal sensitivity pattern.

For survey systems where any four electrodes in four different lines can be used at the same time, another array type that has electrodes along four different lines can be included in the comprehensive data set (Figs $2 \mathrm{e}$ and $\mathrm{j}$ ). The sensitivity patterns of the 'quadruple line' configurations for the alpha and beta types of arrays are shown in Figs 3(e) and 4(e). The sensitivity pattern is similar to the inline arrays (Figs 3a and 4a) except that the high sensitivity values have a diagonal orientation in the area between the four lines. The sensitivity patterns for the equatorial dipole-dipole array type show large positive sensitivity values in the central region between the $A-B$ current and $M-N$ potential dipole pairs (Fig. 5), with regions of large negative values between the $A-M$ and $B-N$ current -potential pairs.

In the array optimization algorithm used by Loke et al. (2010a) for 2-D survey lines, for arrays that are not symmetrical about the midpoint of the survey line the corresponding array configuration on the other half of the survey line is also automatically included in the optimized data set. For a 3-D survey using a rectangular grid of electrodes, there is a four way symmetry compared to a two-way symmetry for a 2-D survey line. Thus a check is included to ensure the symmetrical variations of an array in the three other quadrants of the grid are added to the optimized data set. This can result in the optimized data set having up to three additional arrays over the target number of data points in some cases.

\section{RESULTS}

The results are first presented in terms of the model resolution sections for the conventional arrays and optimized data sets. This is followed by tests using a synthetic model and an experimental tank data set.

\subsection{Model resolution sections}

In this section the model resolution values for surveys carried out using conventional arrays and the optimized arrays are shown. We consider a $21 \mathrm{~m} \times 15 \mathrm{~m}$ survey grid with an electrode spacing of 1 $\mathrm{m}$ in the $x$ direction and line spacing of $2 \mathrm{~m}$ in the $y$ direction. It is a common practice in many $3-\mathrm{D}$ field surveys to use a line spacing of about twice (or more) the inline electrode spacing (Bentley \& Gharibi 2004; Johansson et al. 2007; Rosqvist et al. 2010). The electrodes are arranged along eight parallel lines with 21 electrodes along each line, giving a total of 168 electrode positions. It is first assumed the survey was carried out using the configuration shown in Fig. 1 that imposes limitations on the possible measurements in the $y$ direction using arrays with four electrodes. Only the electrodes along three adjacent lines can be accessed simultaneously. The sides of the model used to calculate the resolution values are extended $4 \mathrm{~m}$ in both the $x$ and $y$ directions beyond the edges of the survey grid. This is to ensure that all the regions of the subsurface that have significant resolution values are included (Loke et al. 2014a). The subsurface is subdivided into model cells with widths of $1 \mathrm{~m}$ in both the $x$ and $y$ directions. The thickness of the first layer is set at $0.5 \mathrm{~m}$, and the thickness of each deeper layer is increased by 5 percent. A total of nine layers are used with the deepest layer at a depth of about $5.5 \mathrm{~m}$ (or about one-quarter the line length in the $x$ direction), giving a total of 5544 model cells. We use a maximum geometric factor of $2261.9 \mathrm{~m}$. (corresponding to a dipole-dipole array with ' $a$ ' spacing of $1 \mathrm{~m}$ and ' $n$ ' value of 8 ) and geometric factor relative error of $5 \mathrm{~m}^{-1}$. The comprehensive data set for this survey grid, which consists of the possible inline alpha and beta arrays as well as their offset and triple line versions, has 538077 data points. The model resolution values for the comprehensive data set are shown in Fig. 6(a) in the form of horizontal sections for the first four layers and a vertical section in the $x-z$ plane across the middle of the survey area. The regions with significant resolution values (above 0.05) extend until the sixth layer (Fig. 6a) with a mean depth of $3.1 \mathrm{~m}$.

Next we consider a data set consisting of all the possible inline (along the $x$ direction) Wenner-Schlumberger (Pazdirek \& Blaha 1996) and dipole-dipole arrays. This gives a total of 3152 data points. The model resolution sections for this data set (Fig. 6b) have significantly lower resolution values. This is partly because the inline arrays have lower sensitivity values for the regions between the lines. The 'Compare R' method is then used to generate a set of 3153 optimized arrays using a 5 percent step size (Loke et al. 2010b). The optimized data set has one extra data point due to the symmetry requirement for the arrays generated. The model 
(a) Comprehensive data set (538077 data points), average resolution 0.200

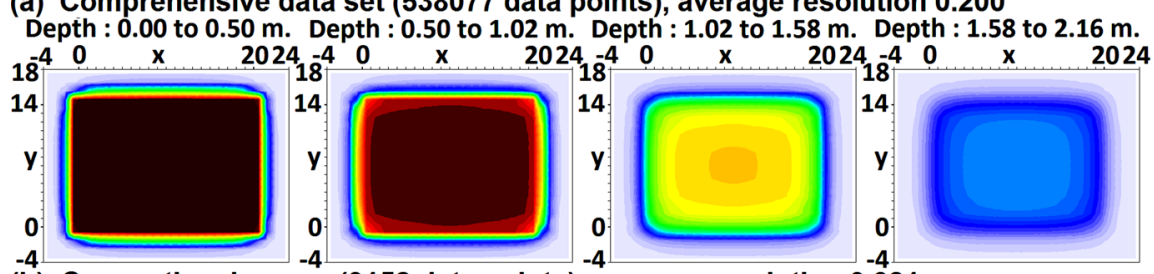

(b) Conventional arrays (3152 data points), average resolution 0.081

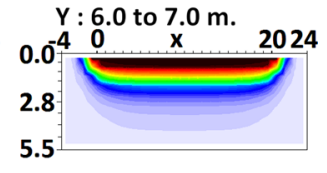

Depth : 0.00 to $0.50 \mathrm{~m}$. Depth : 0.50 to $1.02 \mathrm{~m}$. Depth : 1.02 to $1.58 \mathrm{~m}$. Depth : 1.58 to $2.16 \mathrm{~m}$.

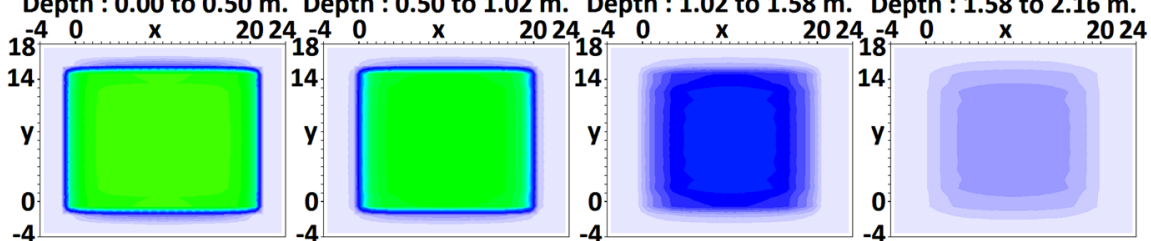

(c) Optimized arrays (3153 data points), average resolution 0.143

Depth : 0.00 to $0.50 \mathrm{~m}$. Depth : 0.50 to $1.02 \mathrm{~m}$. Depth : 1.02 to $1.58 \mathrm{~m}$. Depth : 1.58 to $2.16 \mathrm{~m}$.

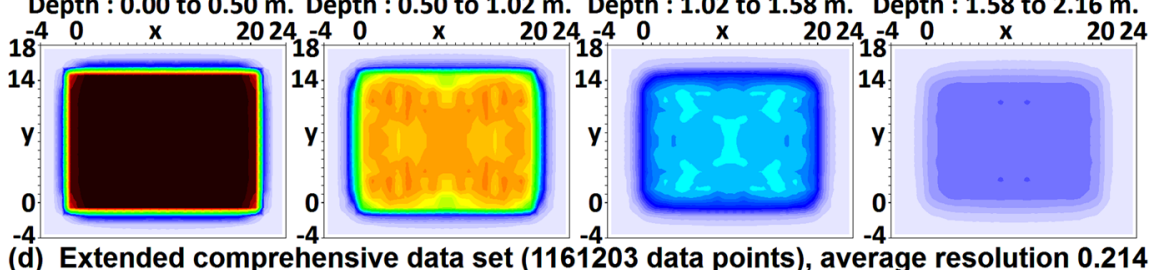

(d) Extended comprehensive data set (1161203 data points), average resolution 0.214
Depth : 0.00 to $0.50 \mathrm{~m}$. Depth : 0.50 to $1.02 \mathrm{~m}$. Depth : 1.02 to $1.58 \mathrm{~m}$. Depth : 1.58 to $2.16 \mathrm{~m}$.

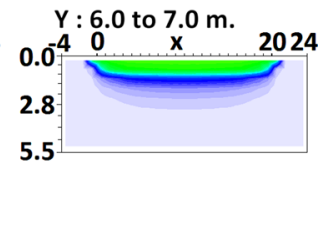

Depth : 0.00 to $0.50 \mathrm{~m}$. Depth : 0.50 to $1.02 \mathrm{~m}$. Depth : 1.02 to $1.58 \mathrm{~m}$. Depth : 1.58 to $2.16 \mathrm{~m}$.

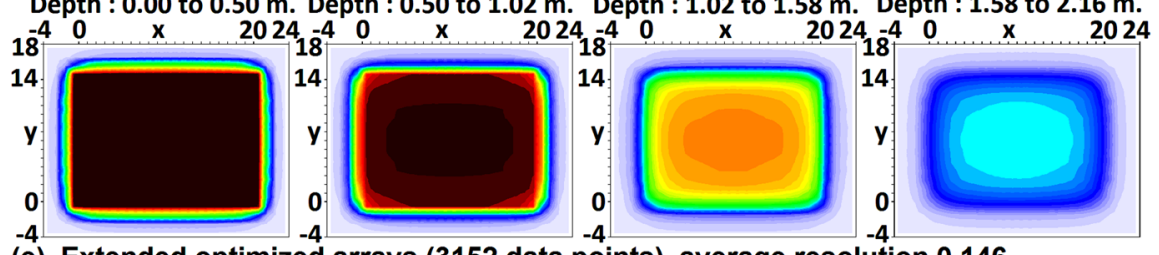

(e) Extended optimized arrays (3152 data points), average resolution 0.146

Depth : 0.00 to $0.50 \mathrm{~m}$. Depth : 0.50 to $1.02 \mathrm{~m}$. Depth : 1.02 to $1.58 \mathrm{~m}$. Depth : 1.58 to $2.16 \mathrm{~m}$.
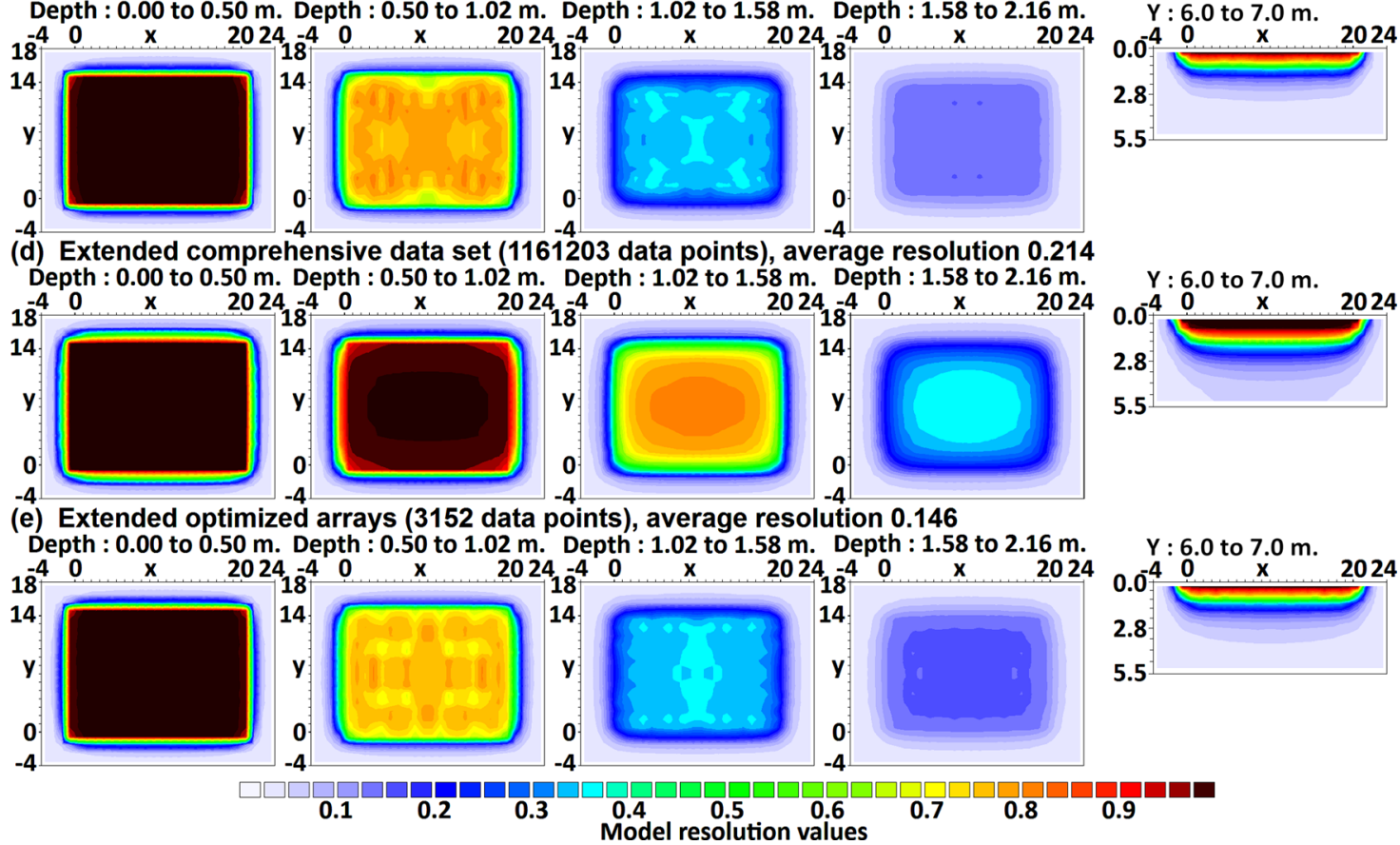

5.5

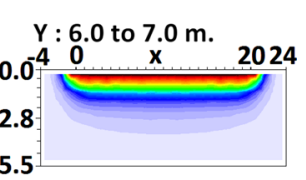

Figure 6. Horizontal and vertical model resolution sections for (a) comprehensive data set, (b) conventional (combined dipole-dipole and WennerSchlumberger) arrays data set with 3152 data points, (c) optimized data set with 3153 data points, (d) 'extended' comprehensive data set that includes arrays that cross more than three survey lines and (e) 'extended' optimized data set with 3152 data points. The top four horizontal slices are shown to illustrate the variations in the $x$ and $y$ directions, while a vertical $x-z$ slice passing through the centre of the model is used to show the vertical variations.

resolution sections for this data set (Fig. 6c) have significantly higher values compared to the conventional arrays data set in all the layers. The region with resolution values of over 0.05 extends to the fifth layer with a mean depth of $2.5 \mathrm{~m}$, compared to the conventional arrays data set where it only extends to the fourth layer with a mean depth of $2.5 \mathrm{~m}$. The average resolution of 0.143 achieved by the optimized data set is significantly higher than that of the conventional arrays data set $(0.081)$. It is about 70 percent of that achieved by the comprehensive data set $(0.200)$ although the number of data points is only 0.6 per cent of that in the comprehensive data set.

We next consider the case where all the electrodes in the survey grid can be accessed at the same time. This allows the array used to traverse across the entire grid instead of being limited to three adjacent lines. Such a system has been developed for special purposes, such as long-term monitoring of hydraulic processes and soil movement (Merrit et al. 2013; Chambers et al. 2014). This allows the use of the 'quadruple line' configurations, as well as the other configurations, with the electrodes spread over several lines (Fig. 7). It also allows the use of the same array configuration oriented in the $y$-direction. The comprehensive data set for the same $21 \times 15$ grid, where any four electrodes within the grid can be accessed at the same time, has 1161203 arrays that is about twice that of the earlier more 'restricted' comprehensive set where the electrodes in an array are confined to three adjacent lines. The model resolution section for this 'extended' comprehensive data set is shown in Fig. 6(d). Although the average resolution (0.214) for the 'extended' comprehensive data set is less than 10 per cent higher than the 'restricted' comprehensive set $(0.200)$, the resolution values at the deeper layers are significantly higher. The regions with significant resolution values extend to the seventh layer (Fig. 6d) with a mean depth of $3.7 \mathrm{~m}$. The optimized data set with 3152 data points generated from the 'extended' comprehensive data set also has significantly higher resolution values in the fourth to sixth layers (Fig. 6e) than the set 
(a)

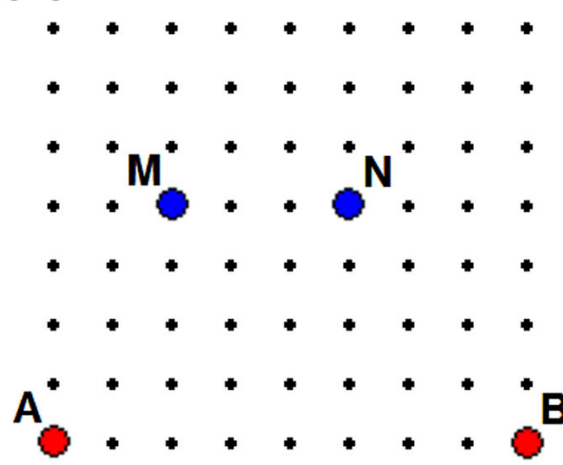

(b)

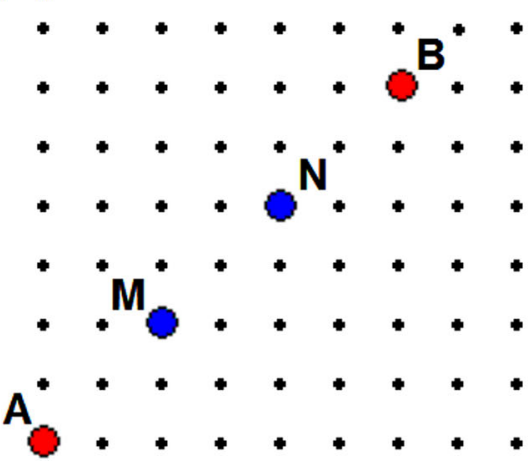

(c)

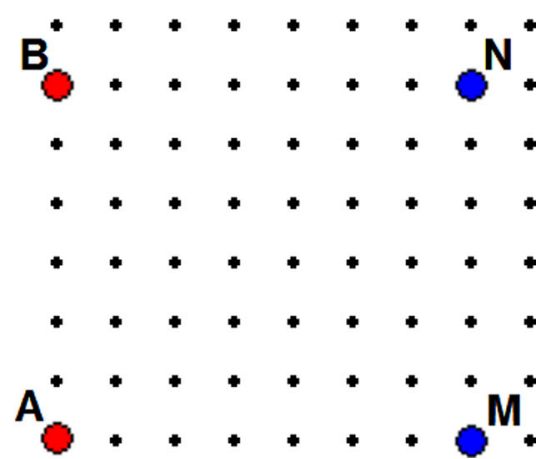

Figure 7. Examples of the (a) double line, (b) quadruple line and (c) equatorial dipole-dipole array types that spread across multiple survey lines in the measurement grid. The small black dots are the positions of the electrodes in the grid.

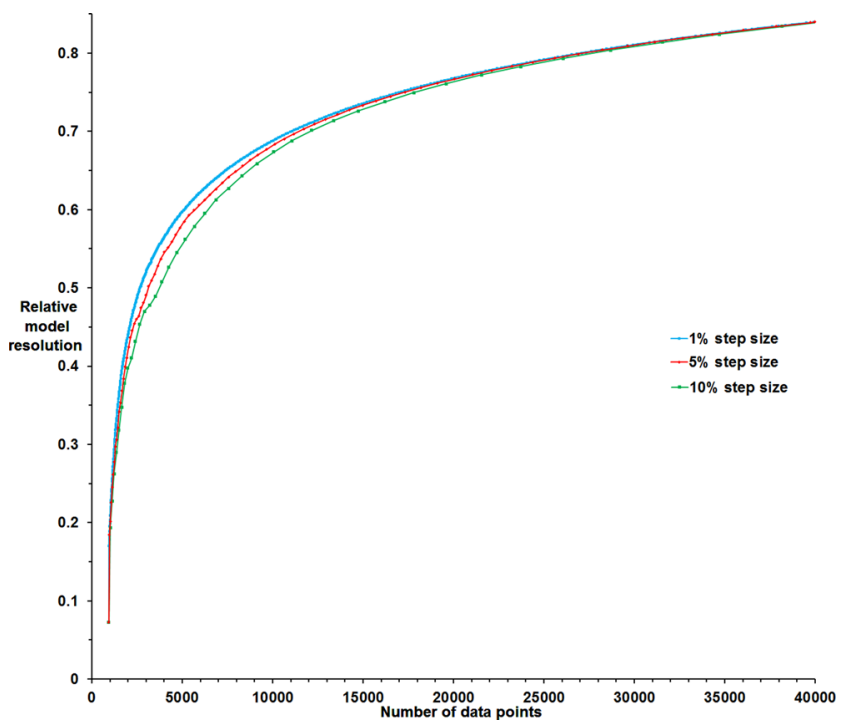

Figure 8. Change of the relative model resolution with the number of data points. The survey grid has 21 electrodes with a spacing of $1 \mathrm{~m}$ in the $x$-direction, and a spacing of $2 \mathrm{~m}$ between eight lines in the $y$-direction. The per cent step size is the percentage increase in the size of the optimized data set after each iteration.

with the 'restricted' comprehensive set (Fig. 6c). This suggests that the arrays generated from the 'extended' comprehensive set have a deeper depth of investigation.

Fig. 8 shows the change of the relative model resolution $S_{r}$, given below in eq. (11), with the number of data points for the optimized data set (generated from the 'restricted' comprehensive set) using different step sizes to increase the size of the optimized data set after each iteration.

$S_{r}=\frac{1}{m} \sum_{j=1}^{j=m} \frac{R_{b}(j, j)}{R_{c}(j, j)}$.

The optimized data set generated using a smaller step size generally has a higher resolution value for the same number of data points, at the expense of a longer calculation time. This is similar to that obtained by Loke et al. (2010b) for 2-D surveys. To generate 40000 data points, the algorithms with the 1,5 and 10 percent step sizes take 42556,8969 and 4739 s, respectively on a computer system with an Intel Sandy Bridge-E 3930 hex-core CPU and a Nvidia 670GTX graphics card. The relative model resolution rises rapidly until about 20000 data points after which it increases slowly. Beyond 30000 data points, the three curves converge to almost identical values.

\subsection{Synthetic model test for a $16 \times 6$ electrodes survey grid}

The test model consists of three rectangular blocks embedded in a two-layer medium (Fig. 9). The electrodes are arranged along 6 lines at $20 \mathrm{~m}$ apart with 16 electrodes $10 \mathrm{~m}$ apart along each line, giving a total of 96 electrodes positions. We set the maximum geometric factor at $22620 \mathrm{~m}$ (corresponding to a dipole-dipole array with $a=10 \mathrm{~m}$ and $n=8$ ). The conventional arrays data set, consisting of all the possible inline Wenner-Schlumberger and dipole-dipole arrays along the $x$ direction, has 1218 data points. In this test, we generate the optimized data sets where the electrodes in an array are restricted to three adjacent lines as well the case where the electrodes can be on any line. The comprehensive data sets for the 'restricted' and 'extended' optimized data sets have 88428 and 141602 arrays, respectively. A step size of 1 percent was used in the array optimization algorithm to generate the arrays (Loke et al. 2010b). Three optimized data sets were generated. The first two optimized data sets have a similar number of data points as the conventional arrays. We generate the arrays for both the 'restricted' and the 'extended' optimized data sets. This was done to investigate the possible improvement that can be obtained by using the additional configurations available in the 'extended' optimized set. The fourth and larger set (from the 'extended' optimized set) has 2500 points to study the improvement in the model resolution with more data points. Voltage-dependent Gaussian random noise (Press et al. 1992; Zhou \& Dahlin 2003) with a mean amplitude of $0.4 \mathrm{~m} \Omega$ was added to the data sets before they were converted into apparent resistivity values. The data sets have a minimum resistance value of about $4.2 \mathrm{~m} \Omega$. Thus, the data points with the lowest resistance values have a noise level of nearly 10 percent. The average noise level is about 0.8 per cent for the conventional arrays data set and 1.5 percent for the optimized data sets. The optimized data sets have a higher noise level due to a higher average geometric factor of the arrays generated compared to conventional arrays (Loke et al. 2010a). The L1-norm method was used for both the data misfit and model roughness (Farquharson \& Oldenburg 1998; Loke et al. 2003) in inversion of the data. A large value of about 0.10 is initially used for the damping factor in eq. (1), which is then adjusted after each iteration by the L-curve method (Farquharson \& Oldenburg 2004; 

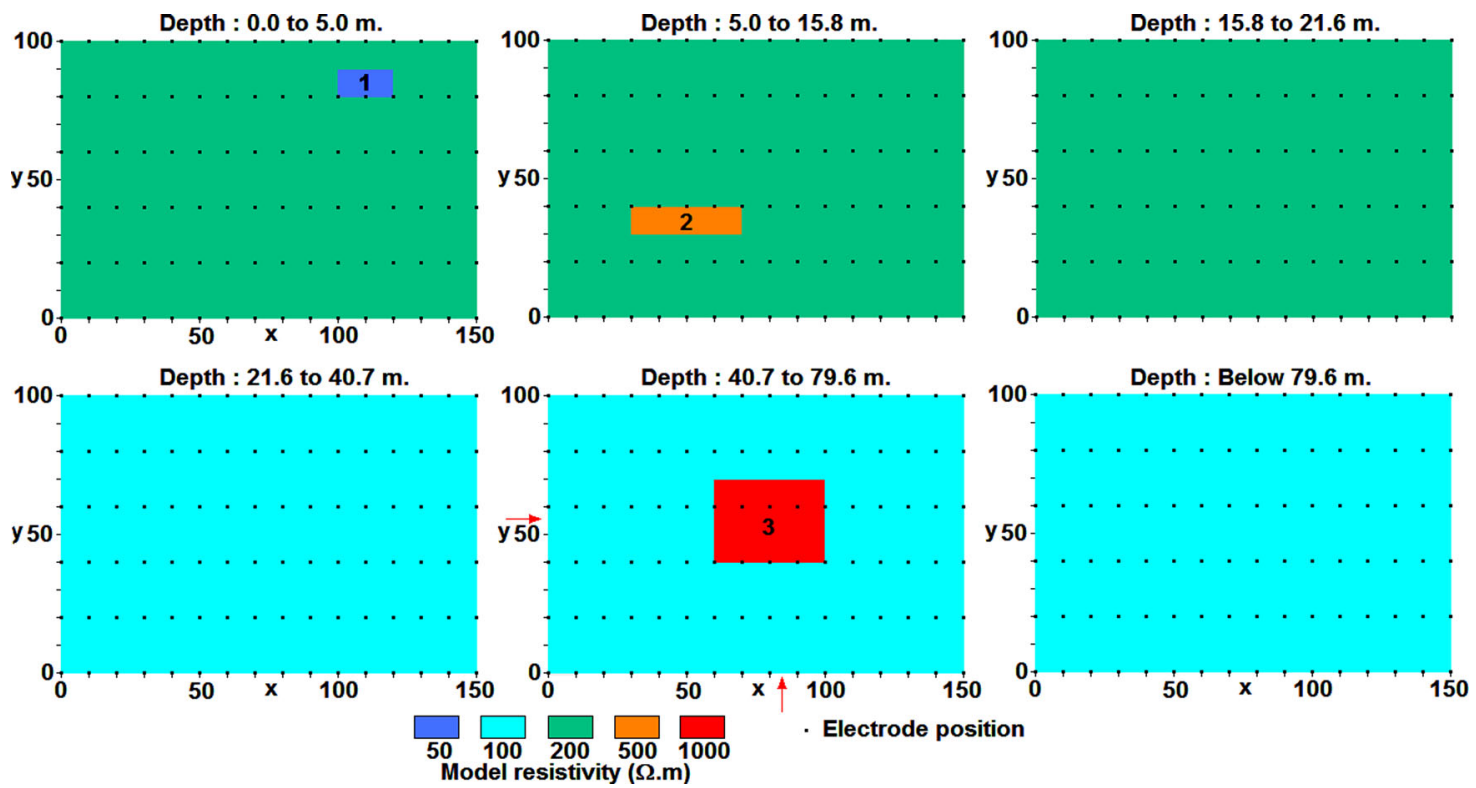

Figure 9. Synthetic test model with three rectangular blocks in a two-layer medium shown in the form of horizontal slices. The positions of the electrodes along the survey lines are marked by black dots. The red arrows at the sides of the horizontal model slice with block 3 show the positions of the inversion model vertical planes shown in Fig. 11.

Loke et al. 2014b) until it reaches an almost constant value (usually within six to eight iterations).

Fig. 10 shows the top five horizontal slices of the inversion model that better illustrates the two blocks in the upper layer. The two blocks are less well resolved by the conventional arrays data set compared to the optimized data sets. The widths of blocks (in the $y$ direction) in the conventional arrays inversion model is twice the true width, while the optimized arrays models show the correct width (Fig. 10). The optimized arrays models give a minimum resistivity value within 2 per cent of the true value of $50 \Omega \mathrm{m}$ for the topmost block while the conventional arrays model give a significantly higher value of $77 \Omega \mathrm{m}$ (Table 1 ). The optimized arrays models also achieve a higher maximum resistivity value of about $400 \Omega \mathrm{m}$ for the deeper $500 \Omega \mathrm{m}$ block, compared to about $300 \Omega \mathrm{m}$ for the conventional arrays model.

All the data sets correctly position the boundary between the two layers at a depth of $2.16 \mathrm{~m}$ (Fig. 11). The resistivity values in the layers (outside the immediate vicinity of the blocks) are generally within 10 per cent of the true values of 200 and $100 \Omega \mathrm{m}$ for all the models, except near the edges of the models where there is not much data. Fig. 11 shows vertical slices of the models that pass through the centre of the $1000 \Omega \mathrm{m}$ high-resistivity block (Fig. 9). The conventional arrays model fails to resolve the block. The 'restricted' and smaller 'extended' optimized data sets do show a region with significantly higher resistivity values at the location of the block (Figs $11 \mathrm{~b}$ and c). It is more clearly resolved in the large 'extended' optimized data set (Fig. 11d) that gives a maximum value of 145 $\Omega \mathrm{m}$ (Table 1) that is well above the background value of $100 \Omega \mathrm{m}$.

To quantitatively determine the differences in the accuracy of the models, we calculate the average absolute model misfits between the true $\left(r_{t}\right)$ and the calculated $\left(r_{c}\right)$ model values using the following equation.

$\delta_{m}=\frac{1}{n} \sum_{i=1}^{n}\left|\log \left(r_{t}(i)\right)-\log \left(r_{c}(i)\right)\right|$.

The average model misfits of the conventional arrays, 'restricted' optimized, small and large 'extended' optimized data sets are 0.159 ,
$0.147,0.146$ and 0.139 (Table 1). The optimized data sets models have significantly lower model misfits compared to the conventional arrays model, while the large optimized data set model is significantly more accurate. Although the data misfits for the optimized arrays models (1.4-1.6 per cent) are about twice that of the conventional arrays model $(0.8$ percent), the inversion models are more accurate.

\subsection{Experimental data set}

In this section, we show the results from an experimental data set measured in a tank using the $16 \times 6$ electrodes survey grid layout described in the previous section. However, the inline spacing between the electrodes is scaled down to $0.05 \mathrm{~m}(5 \mathrm{~cm})$. The tank has dimensions of $1.4 \mathrm{~m} \times 0.8 \mathrm{~m} \times 1.0 \mathrm{~m}$. The tank was filled with water with resistivity of about $13.8 \Omega \mathrm{m}$ to a depth of $0.995 \mathrm{~m}$, and three rectangular plastic blocks were placed at different depths in the water (Fig. 12). The sides of the tank are $0.325 \mathrm{~m}$ from the ends of the lines in the $x$ direction, and $0.15 \mathrm{~m}$ from the first and last lines in the $y$ direction. Since the distance of the electrodes at the ends of the grid from the tank sides is less than the extent of the grid in the $x$ direction $(0.75 \mathrm{~m})$, the tank boundaries have a significant effect on the measured potentials. We set the maximum geometric factor at $343.1 \mathrm{~m}$ (corresponding to a dipole-dipole array with $a=0.05 \mathrm{~m}$ and $n=12$ ). Initially measurements were made using optimized arrays generated using a homogeneous half-space model. The conventional arrays gave reasonable apparent resistivity values but it was found that some of the optimized arrays gave values that were much higher or lower. Some of the optimized array configurations even gave negative apparent resistivity values. This was caused by the sides of the tank that appear as boundaries with very large resistivity contrasts. The areas with large sensitivity values for the conventional inline arrays are mainly confined near the array axis, so they are not as sensitive to the sides of the tank. However, the offset versions used in the optimized arrays, particularly for the beta array configuration (Fig. 4), have comparatively larger sensitivity values away from the electrode positions. The geometric 
(a) Conventional arrays data set (1218 data points), data misfit $=0.8 \%$
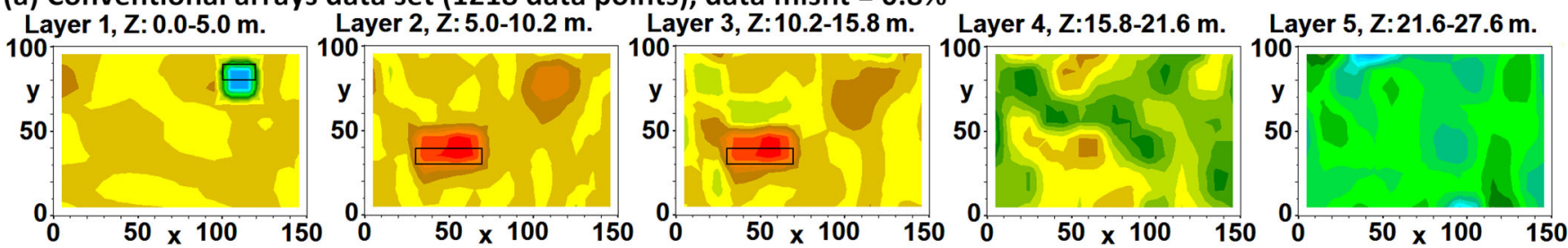

(b) Restricted optimized data set (1219 data points), data misfit $=1.4 \%$
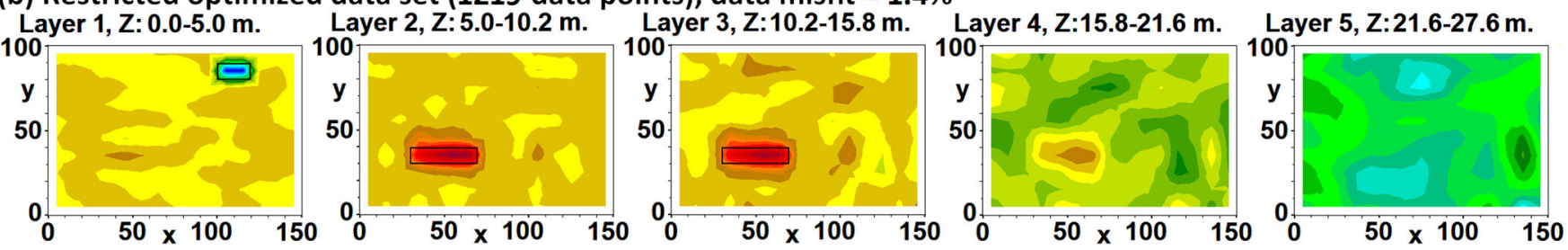

(c) Extended optimized data set (1218 data points), data misfit $=1.4 \%$
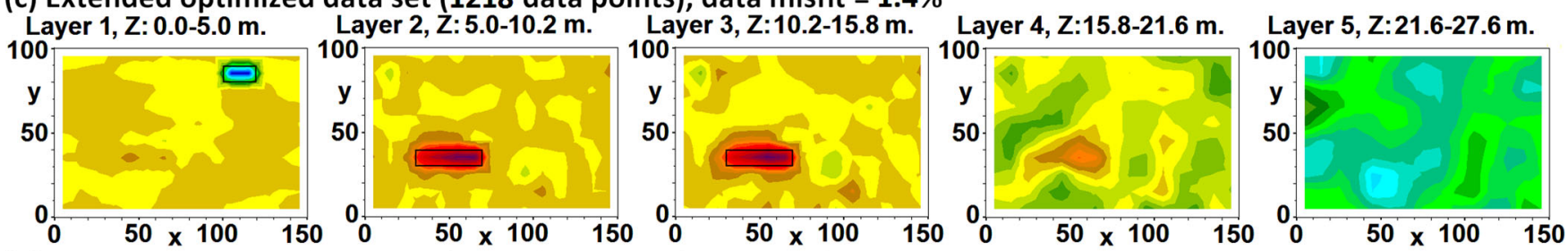

(d) Extended optimized data set ( 2500 data points), data misfit $=1.6 \%$
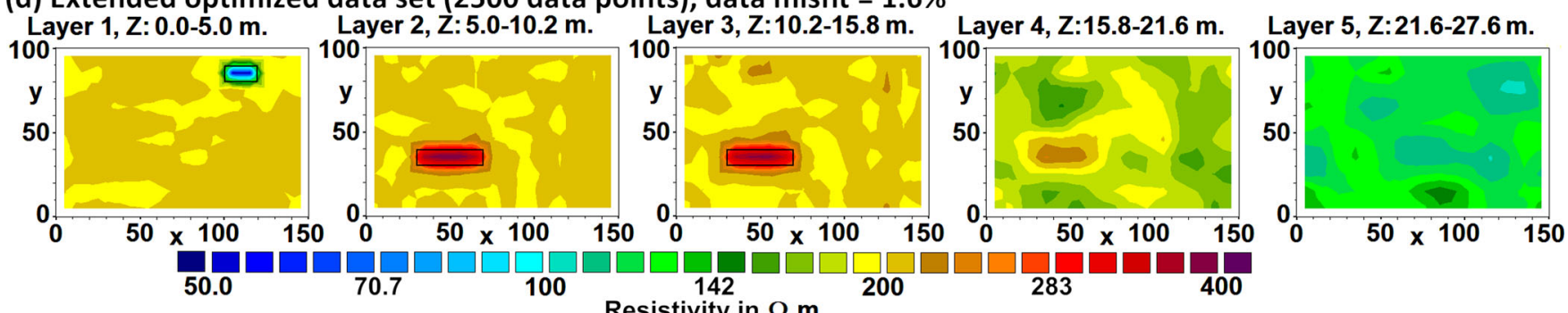

Figure 10. First five layers of inversion models for (a) conventional arrays data set (1218 data points), (b) restricted optimized data set (1219 data points), (c) extended optimized data set (1218 data points) and (d) large extended optimized data set (2500 data points). The actual positions of the two blocks in the top layer are marked by black rectangles. The boundary between the two layers is at the top of layer 5 .

Table 1. Maximum resistivity values (in $\Omega \mathrm{m}$ ) achieved by the inversion models for the different data sets at the positions of the blocks 2 and 3 in the synthetic model, together with the overall model misfit. The minimum resistivity value is shown for block 1 . True resistivity values at blocks 1,2 and 3 are 50,500 and $1000 \Omega \mathrm{m}$, respectively.

\begin{tabular}{lcccc}
\hline Data set & Block 1 & Block 2 & Block 3 & Model misfit \\
\hline Conventional arrays 1218 arrays & 77 & 296 & 124 & 0.159 \\
Restricted optimized 1219 arrays & 51 & 380 & 131 & 0.147 \\
Extended optimized 1218 arrays & 50 & 411 & 129 & 0.146 \\
Extended optimized 2500 arrays & 49 & 396 & 145 & 0.139 \\
\hline
\end{tabular}

factor relative error values calculated for a homogeneous half-space that were used to filter out the unstable arrays are then not sufficiently accurate since they do not take into account the effect of the sides of the tank. To obtain more accurate values for the geometric factor relative error for the optimized arrays within the tank, we calculate them numerically using a 3-D finite-difference program (Dey \& Morrison 1979). A tank model filled with a homogenous medium is used. The details on the finite-difference method used are given in the Appendix. By using the numerically calculated geometric factor relative errors values to filter out the unstable arrays, it was found that the optimized arrays generated gave reasonable apparent resistivity values of between 7 and $25 \Omega \mathrm{m}$. The Jacobian matrix used in the calculation of the optimized arrays was also generated using the finite-difference program so that it includes the effect of the tank walls. Data were measured for the redesigned optimized arrays and the conventional arrays using a GEOTOM Geolog2000 single channel resistivity meter capable of addressing up to 100 electrodes. Reciprocal measurements were also made for each array, and the difference between each reciprocal pair of values was used to calculate the standard error in the mean for each 
(a) Conventional arrays data set (1218 data points), data misfit $=0.8 \%$
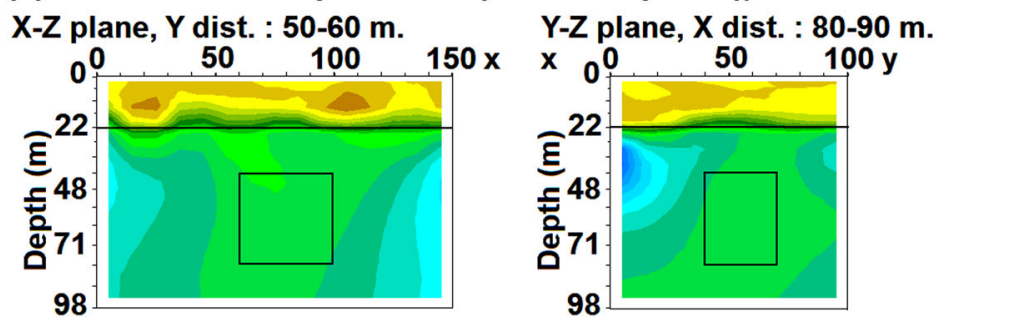

(b) Restricted optimized data set (1219 data points), data misfit $=1.4 \%$

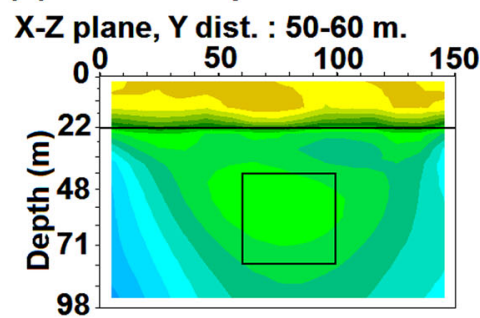

Y-Z plane, $X$ dist. : $80-90 \mathrm{~m}$.

(c) Extended optimized data set (1218 data points), data misfit $=1.4 \%$
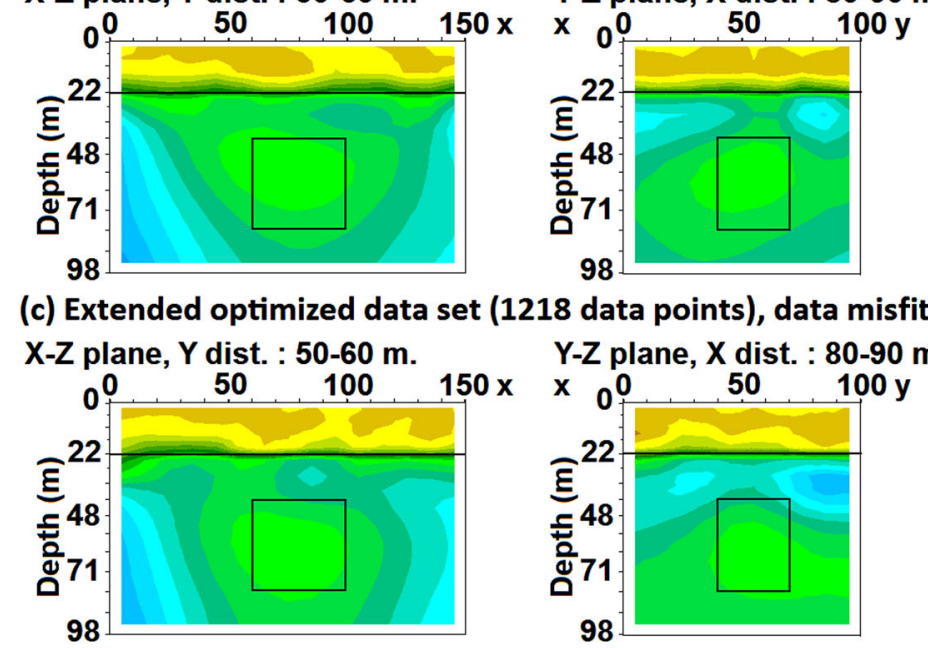

(d) Extended optimized data set (2500 data points), data misfit $=1.6 \%$

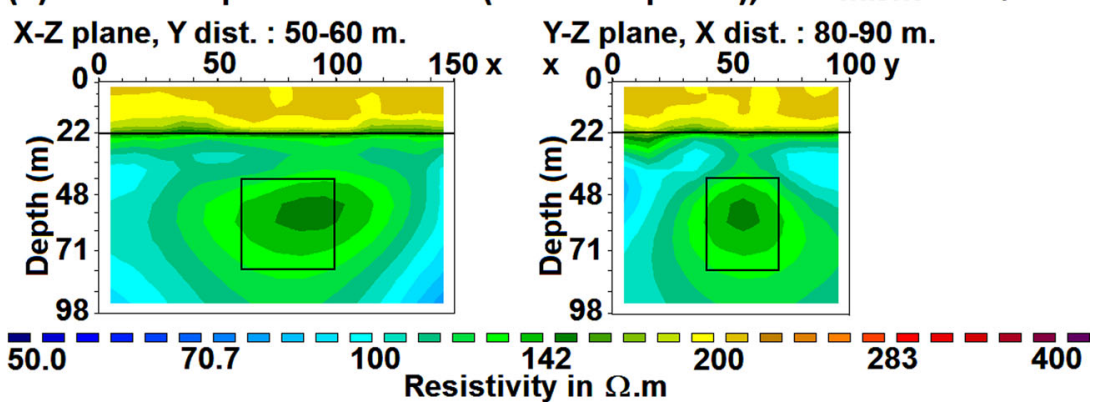

Figure 11. Inversion models shown in the form of vertical slices through the middle of the bottom high-resistivity block. (a) Conventional arrays, (b) restricted optimized arrays, (c) extended optimized arrays and (d) large extended optimized data set with 2500 data points. The actual position of the block is marked by a black rectangle, and the boundary between the two layers is marked by a black line.

measurement, referred to as the reciprocal error. Data quality was very high; for the conventional arrays, 97.1 per cent of the data had reciprocal errors $<1$ percent. The corresponding fractions of the optimized data sets were $94.8,94.7$ and 94.3 per cent for the arrays with 1416, 2500 and 5001 measurements, respectively.

Fig. 13 shows the inversion models for the conventional arrays data set (Wenner-Schlumberger and dipole-dipole arrays with 1414 data points), as well as the optimized data sets with 1416, 2500 and 5001 data points. The inversion model consists of 20 layers with a total of 6720 cells. The models are shown as 3-D plots due to the large number of cells and the fairly simple homogenous background medium. In this plot, the cells with resistivity values of above $35.5 \Omega \mathrm{m}$ in the vicinity of blocks A and B are shown. For block C, we highlight model cells with resistivity values of above $17.8 \Omega \mathrm{m}$ as the resolution decreases with depth. In the conventional arrays model (Fig. 13a), the top two blocks (A and B) have widths of about twice the true width. This is similar to the result obtained for the near surface high-resistivity blocks (Fig. 10a) in the synthetic model test. The deepest block (C) appears as a region with slightly higher resistivity values with a maximum value of $17.9 \Omega \mathrm{m}$ (Table 2 ) near the top of the actual block position (Fig. 13a). This maximum value is significantly lower than the value of $19.4 \Omega \mathrm{m}$ achieved by the optimized data set (Fig. 13b, Table 2). The model for the optimized data set also exhibits higher resistivities for blocks A and B, as well as resolving their widths correctly. The main effect of increasing the number of data points in the optimized data set to 2500 and 5001 data points is an increase in the model resistivity at the locations of the three blocks (Figs 13c and d, Table 2). The region with resistivity values above $17.8 \Omega \mathrm{m}$ also covers the entire volume of block C. Pearson correlation coefficients between the inverted images and the model were also calculated to quantify image quality (Wilkinson et al. 2012). Since the model targets are infinitely resistive, the 


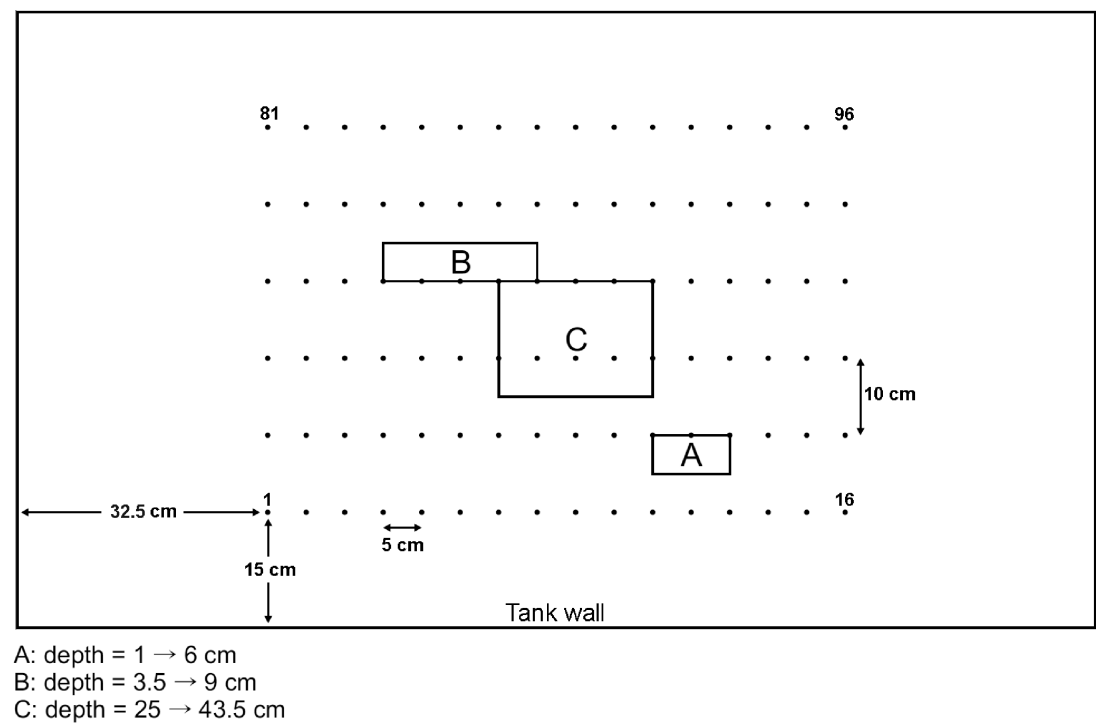

Figure 12. Tank layout with positions of boundaries (outer rectangle), electrodes (dots) and plastic rectangular blocks (inner rectangles). The inline electrode spacing is $5 \mathrm{~cm}$ while the distance between the lines is $10 \mathrm{~cm}$. Note A is the topmost block while $\mathrm{C}$ is the deepest block.

resistivities $\rho$, of the model and images were transformed into a coefficient given by

$r=\frac{\rho-\rho_{0}}{\rho+\rho_{0}}$

where $r=1.0$ corresponds to the infinite target resistivity and $r=0.0$ corresponds to the resistivity of the water $\left(\rho_{0}=13.8\right.$ $\Omega \mathrm{m})$. The correlation coefficients are then calculated between the model and images in terms of $r$ (Table 2). They indicate that a significant improvement in image quality is obtained by using the optimized 1416 data set instead of the conventional arrays. A further increase of similar magnitude is gained by increasing the size of the optimized set to 2500 data points. However, going to 5001 measurements produces only modest increases in the target's contrasts, and therefore does not improve the correlation coefficient much further.

The models for the optimized data sets have slightly higher data misfits compared to the conventional arrays data set. This is expected as the optimization procedure tends to choose arrays with higher geometric factors (Wilkinson et al. 2012). However, despite the higher data misfits, the optimized data sets have better resolution, particularly at the deepest block. Note that the depth of $0.45 \mathrm{~m}$ to the base of the deepest block is more than half the length of the lines (in the $x$ direction) of $0.75 \mathrm{~m}$. Despite being a challenging target, it is reasonably well resolved by the largest optimized data set. The optimization procedure provides an automatic and convenient means to generate the most suitable arrays for a 3-D survey. It takes about $197 \mathrm{~min}$ to generate the optimized data set with 5001 data points using the single-step algorithm (Loke et al. 2010b) on the computer system used.

\section{CONCLUSION}

A modification of the algorithm used to generate the viable arrays for 2-D surveys to include offset versions of the alpha and beta arrays is presented for 3-D surveys. The geometric factor relative error, together with the geometric factor, was used to filter out the unstable arrays in generating the comprehensive data set. Tests with synthetic models and an experimental data set show that the optimized arrays perform significantly better than conventional arrays.

The results from this study show that by using offset versions of the conventional four-electrode arrays in a 3-D survey, the resolution of the inversion models can be significantly increased (particularly in the deeper sections of the models) using the same number of data points compared to the use of only conventional arrays. A significant improvement is obtained even in situations where the electrodes in an array are confined to three adjacent lines, such as with the ABEM SAS resistivity meter system. However, if the electrodes in an array can occupy any position in the measurement grid, the model resolution can be further improved. Increasing the number of data points used in the optimized arrays does increase the model resolution, but the incremental benefit of adding more measurements decreases beyond a certain limit (about 2 per cent of the comprehensive data set).

Research is currently being carried on using the 3-D array optimization algorithm for surveys with subsurface electrodes using conventional vertical boreholes (Wilkinson et al. 2008), as well as along horizontal lines of subsurface electrodes (Harro \& Kruse 2013). As these surveys involve electrodes on different lines, it will also be necessary to use the geometric factor relative error to filter out potentially unstable arrays. The use of optimized versions of the pole-dipole array to obtain a deeper depth of investigation (White et al. 2001, 2003; Blome et al. 2011) is also being investigated. Modifications to the array optimization routine to accommodate the special requirements for I.P. (Induced Polarization) surveys (such as reducing EM coupling and telluric noise) are also being developed.

\section{ACKNOWLEDGEMENTS}

This paper is published with the permission of the Executive Director of the British Geological Survey (NERC). We would like to thank two anonymous reviewers for their useful comments that have helped to improve the paper. 
(a) Conventional arrays (1414 data points), data misfit 0.5\%

(b) Optimized arrays (1416 data points), data misfit $0.7 \%$
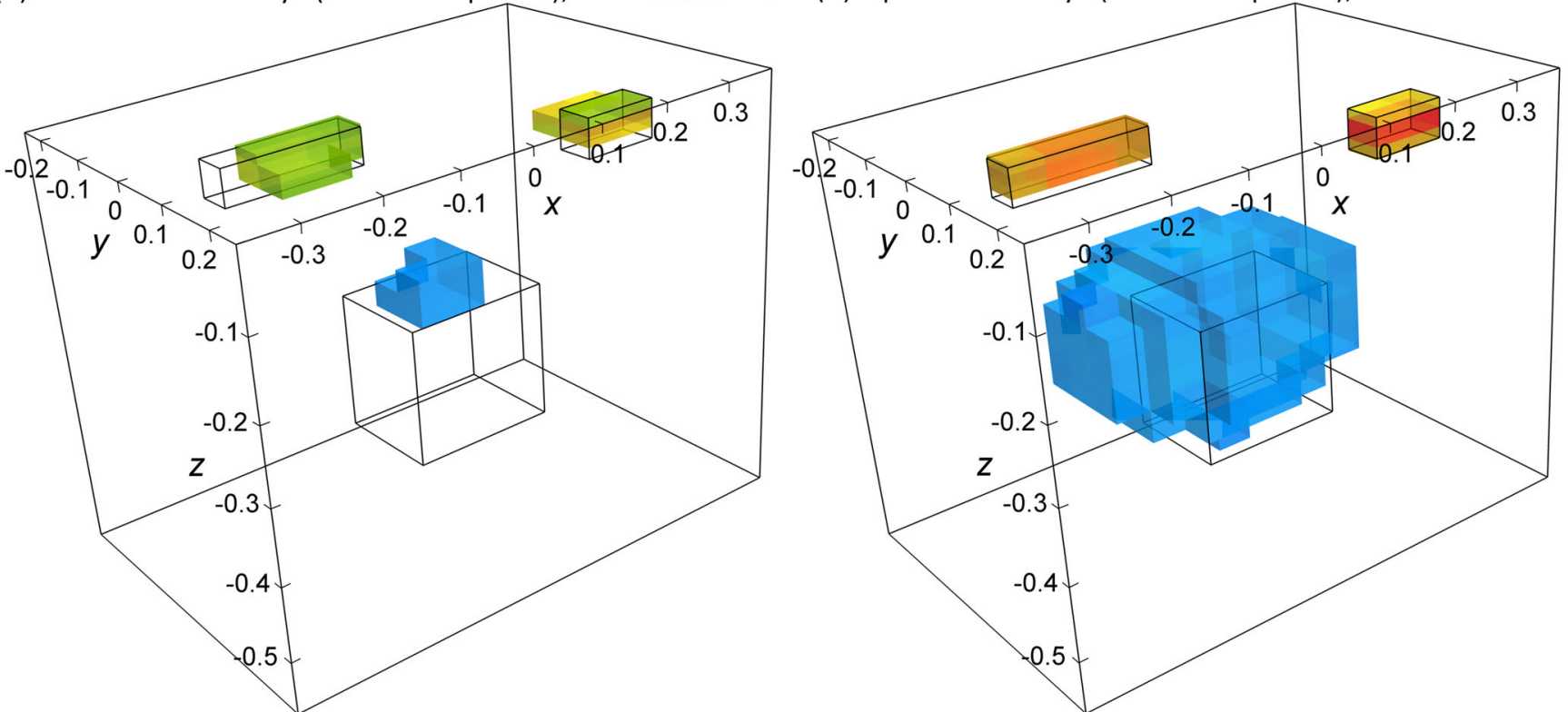

(c) Optimized arrays (2500 data points), data misfit $0.6 \%$

(d) Optimized arrays (5001 data points), data misfit 0.6\%

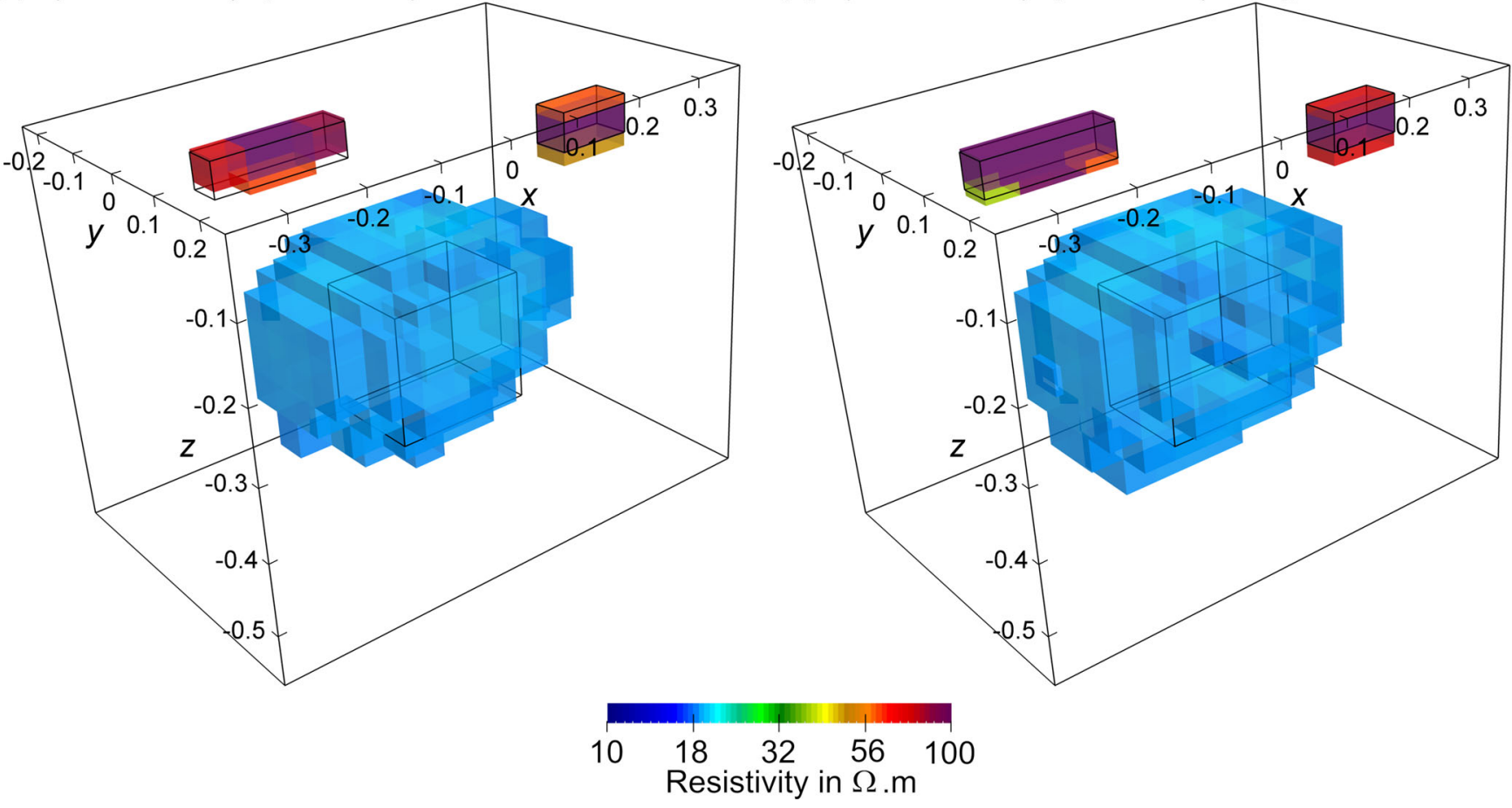

Figure 13. Inversion models (for the tank layout shown in Fig. 12) for (a) conventional arrays data set with 1414 data points, (b) optimized data set with 1416 data points, (c) optimized data set with 2500 data points and (d) optimized data set with 5001 data points. The actual positions of the blocks are marked by prisms with black outlines.

Table 2. Maximum resistivity values achieved by the inversion models for the different tank experiment data sets at the positions of the plastic blocks, together with the overall model correlation coefficients.

\begin{tabular}{lrccc}
\hline Data set & \multicolumn{3}{c}{ Maximum resistivity $(\Omega \mathrm{m})$} & Model correlation \\
& Block A & Block B & Block C & coefficient \\
\hline Conventional arrays & 49.1 & 39.8 & 17.9 & 0.435 \\
Optimized 1416 arrays & 67.7 & 58.2 & 19.4 & 0.514 \\
Optimized 2500 arrays & 132.9 & 134.1 & 20.2 & 0.571 \\
Optimized 5001 arrays & 160.0 & 172.0 & 20.7 & 0.575 \\
\hline
\end{tabular}




\section{REFERENCES}

Auken, E., Pellerin, L., Christensen, N.B. \& Sørensen, K., 2006. A survey of current trends in near-surface electrical and electromagnetic methods, Geophysics, 71, G249-G260.

Bentley, L.R. \& Gharibi, M., 2004. Two- and three-dimensional electrical resistivity imaging at a heterogeneous remediation site, Geophysics, 69, 674-680.

Blome, M., Maurer, H. \& Greenhalgh, S., 2011. Geoelectric experimental design - efficient acquisition and exploitation of complete pole-bipole data sets, Geophysics, 76, F15-F26.

Carpenter, E.W. \& Habberjam, G.M., 1956. A tri-potential method of resistivity prospecting, Geophysics, 11, 455-469.

Chambers, J.E, Loke, M.H., Ogilvy, R.D. \& Meldrum, P.I., 2004. Noninvasive monitoring of DNAPL migration through a saturated porous medium using electrical impedance tomography, J. Contam. Hydrol., 68, $1-22$.

Chambers, J.E., Kuras, O., Meldrum, P.I., Ogilvy, R.O. \& Hollands, J., 2006. Electrical resistivity tomography applied to geologic, hydrogeologic, and engineering investigations at a former waste-disposal site, Geophysics, 71, B231-B239.

Chambers, J.E. et al., 2012. Bedrock detection beneath river terrace deposits using three-dimensional electrical resistivity tomography, Geomorphology, 177-178, 17-25.

Chambers, J.E. et al., 2014. 4D electrical resistivity tomography monitoring of soil moisture dynamics in an operational railway embankment, Near Surf. Geophys., 12, 61-72.

Dahlin, T., 1996. 2D resistivity surveying for environmental and engineering applications, First Break, 14, 275-284.

Dahlin, T. \& Loke, M.H., 1998. Resolution of 2D Wenner resistivity imaging as assessed by numerical modelling, J. appl. Geophys., 38, 237-249.

Dahlin, T., Bernstone, C. \& Loke, M.H., 2002. A 3D resistivity investigation of a contaminated site at Lernacken in Sweden, Geophysics, 60, 16821690.

deGroot-Hedlin, C. \& Constable, S., 1990. Occam's inversion to generate smooth, two-dimensional models from magnetotelluric data, Geophysics, $\mathbf{5 5}, 1613-1624$.

Dey, A. \& Morrison, H.F., 1979. Resistivity modeling for arbitrarily shaped three-dimensional shaped structures, Geophysics, 44, 753-780.

Ellis, R.G. \& Oldenburg, D.W., 1994. Applied geophysical inversion, Geophys. J. Int., 116, 5-11.

Farquharson, C.G. \& Oldenburg, D.W., 1998. Nonlinear inversion using general measures of data misfit and model structure, Geophys. J. Int., 134, 213-227.

Farquharson, C.G. \& Oldenburg, D.W., 2004. A comparison of automatic techniques for estimating the regularization parameter in non-linear inverse problems, Geophys. J. Int., 156, 411-425.

Gharibi, M. \& Bentley, L.R., 2005. Resolution of 3-D electrical resistivity images from inversions of 2-D orthogonal lines, J. Environ. Eng. Geophys., 10, 339-349.

Harro, D. \& Kruse, S., 2013. Improved imaging of covered karst with the multi-electrode resistivity implant technique, in Proceedings of the 13th Sinkhole Conference, NCKRI Symposium 2, Carlsbad, New Mexico, USA, pp. 213-219.

Johansson, B., Jones, S., Dahlin, T. \& Flyhammar, P., 2007. Comparisons of 2D- and 3D-inverted resistivity data as well as of resistivity- and IPsurveys on a landfill, in Proceedings of the Near Surface 2007, Istanbul, Turkey, Expanded Abstracts, P42.

Loke, M.H. \& Barker, R.D., 1996. Practical techniques for 3D resistivity surveys and data inversion, Geophys. Prospect., 44, 499-523.

Loke, M.H., Acworth, I. \& Dahlin, T., 2003. A comparison of smooth and blocky inversion methods in 2D electrical imaging surveys, Explor. Geophys., 34, 182-187.

Loke, M.H., Wilkinson, P.W. \& Chambers, J.E., 2010a. Fast computation of optimized electrode arrays for 2D resistivity surveys, Comput. Geosci., 36, 1414-1426.

Loke, M.H., Wilkinson, P.W. \& Chambers, J.E., 2010b. Parallel computation of optimized arrays for 2-D electrical imaging, Geophys. J. Int., 183, $1202-1315$.
Loke, M.H., Chambers, J.E., Rucker, D.F., Kuras, O. \& Wilkinson, P.B., 2013a. Recent developments in the direct-current geoelectrical imaging method, J. appl. Geophys., 95, 135-156.

Loke, M.H., Chambers, J.E. \& Wilkinson, P.B., 2013b. Computation of Optimized Arrays for 3-D Electrical Imaging Surveys, in Proceedings of the Near Surface Geoscience 2013-19th European Meeting of Environmental and Engineering Geophysics, Bochum, Germany, 9-11 September 2013, We P 22.

Loke, M.H., Wilkinson, P. B., Chambers, P.B. \& Strutt, M., 2014a. Optimized arrays for 2-D cross-borehole electrical tomography surveys, Geophys. Prospect., 62, 172-189.

Loke, M.H., Dahlin, T. \& Rucker, D.F., 2014b. Smoothness-constrained time-lapse inversion of data from 3-D resistivity surveys, Near Surf. Geophys., 12, 5-24.

Maurer, H., Curtis, A. \& Boerner, D.E., 2010. Recent advances in optimized geophysical survey design, Geophysics, 75, 75A177-75A194.

Merritt, A.J. et al., 2013. 3D ground model development for an active landslide in Lias mudrocks using geophysical, remote sensing and geotechnical methods, Landslides, 11, 537-550.

Pazdirek, O. \& Blaha, V., 1996. Examples of resistivity imaging using ME100 resistivity field acquisition system, in Proceedings of the EAGE 58th Conference and Technical Exhibition Extended Abstracts, Amsterdam, P050.

Press, W.H., Teukolsky, S.A., Vetterling, W.T. \& Flannery, B.P., 1992. Numerical Recipes in C, 2nd edn, Cambridge Univ. Press, 994 pp.

Rosqvist, H., Leroux, V., Dahlin, T., Johansson, S. \& Svensson, M., 2010. An evaluation of the potential of the geoelectrical resistivity method for mapping gas migration in landfills, in Proceedings of the SAGEEP 2010, Vol. 1, Keystone, Colorado, pp. 369-378.

Stummer, P., Maurer, H. \& Green, A., 2004. Experimental design: electrical resistivity data sets that provide optimum subsurface information, Geophysics, 69, 120-129.

White, R.M.S., Collins, S., Denne, R., Hee, R. \& Brown, P., 2001. A new survey design for 3D IP modelling at Copper hill, Explor. Geophys., 32, $152-155$.

White, R.M.S., Collins, S. \& Loke, M.H., 2003. Resistivity and IP arrays, optimised for data collection and inversion, Explor. Geophys., 34, 229232.

Wilkinson, P.B., Meldrum, P.I., Chambers, J.E., Kuras, O. \& Ogilvy, R.D., 2006. Improved strategies for the automatic selection of optimized sets of electrical resistivity tomography measurement configurations, Geophys. J. Int., 167, 1119-1126.

Wilkinson, P.B., Chambers, J.E., Lelliott, M., Wealthall, P. \& Ogilvy, R.D., 2008. Extreme sensitivity of crosshole electrical resistivity tomography measurements to geometric errors, Geophys. J. Int., 173, 49-62.

Wilkinson, P.B., Loke, M.H., Meldrum, P.I., Chambers, J.E., Kuras, O., Gunn, D.A. \& Ogilvy, R.D., 2012. Practical aspects of applied optimised survey design for Electrical Resistivity Tomography, Geophys. J. Int., 189, 428-440.

Zhou, B. \& Dahlin, T., 2003. Properties and effects of measurement errors on 2D resistivity imaging surveying, Near Surf. Geophys., 1, 105-117.

\section{APPENDIX: CALCULATION OF THE GEOMETRIC FACTOR RELATIVE ERROR FOR A TANK MODEL}

The calculation of the geometric factor sensitivity values for a homogenous half-space was described earlier in the paper. The values can be calculated analytically as the potential values have a simple mathematical form. However for a non-infinite or nonhomogeneous medium where the potentials due to a current source do not have a simple mathematical form, the effective geometric factor (and associated sensitivity values) have to be calculated numerically (Chambers et al. 2004). To calculate the potentials values, we use the finite-difference method (Dey \& Morrison 1979; Loke \& Barker 1996). To simulate the walls of the tank, the mesh cells 
beyond the tank walls are assigned a very high resistivity value compared to the medium within the tank. We calculated the potential values for the dipole-dipole and Wenner-Schlumberger arrays for a homogenous medium $\left(\rho_{w}\right)$ with a resistivity of about $13.8 \Omega \mathrm{m}$ (from measurements of the water resistivity) within the tank. Tests were conducted using resistivity values ranging from 10 to 10000 times the water resistivity for the material beyond the tank walls. It was found that the potential values did not change significantly beyond a resistivity contrast of about 1000 times. Thus we used this value in the forward modelling routine.

A rectangular mesh in the $x$ and $y$ directions was used for the finite-difference routine. Normally using a mesh with four nodes between adjacent electrode positions give sufficiently accurate results for calculating the potential values (Dey \& Morrison 1979). The relationship between the apparent resistivity $\left(\rho_{a}\right)$, the geometric factor $(K)$ and the potential $(\phi)$ can be written as

$\rho_{a}=K \phi / I$

or

$\rho_{a}=K R$,

where $R$ is the resistance. The above equation can be rearranged as $K=R / \rho_{a}$.

The apparent resistivity $\left(\rho_{a}\right)$ is the same as the true resistivity $\left(\rho_{w}\right)$ for a homogeneous half-space. To calculate the effective geometric factor numerically for a non-infinite medium, we use the ratio of the calculated resistance value (using the finite-difference method) to the resistivity of the homogeneous material within the tank. To calculate the change in the geometric factor due to a change in the electrode position in the $x$-direction, we calculate the resistance value $\left(R_{x}\right)$ using an auxiliary electrode position placed at one node spacing from the normal electrode position. The change in the geometric factor due to a change in the position of the electrode in the $x$-direction is approximated by

$\frac{\partial K}{\partial x} \approx \frac{R_{x}-R}{\rho_{w} \Delta x}$.

The change in the geometric factor due to a change in the electrode position in the $y$-direction can be calculated in a similar way using an auxiliary electrode position that is shifted by one node spacing in this direction. Finally, we calculate the geometric factor sensitivity and relative error using eqs (6) and (9). We first compare the values calculated numerically using the finite-difference program with those calculated analytically for a homogenous halfspace. It was found that the difference between analytically and numerically calculated values increase with the geometric factor relative error value $R_{\mathrm{E}}$. For values of $R_{\mathrm{E}}$ greater than $5 \mathrm{~m}^{-1}$, the difference was greater than 5 percent if a mesh using four nodes between adjacent electrode positions was used. It was necessary to use a mesh with eight nodes between adjacent electrodes positions to obtain sufficiently accurate values for the geometric factor relative error. We note that the geometric factor relative error values are only used to filter out the unstable arrays in the comprehensive data set prior to finding the optimized arrays, and are not used for the calculation of the apparent resistivity values or the Jacobian matrix values in the inversion of the data sets. Thus, the finer mesh with eight nodes was only used for the calculation of the geometric factor relative error values, while for the data inversion we use the standard mesh with four nodes between adjacent electrode positions. 